\title{
Impact of sectoral allocation of foreign aid on gender equity and human development
}

\section{Lynda Pickbourn ${ }^{1}$ and Léonce Ndikumana ${ }^{2}$}

July 2013

\begin{abstract}
While developing countries have made some progress in achieving human development since the turn of the century, many are still lagging behind in important human development goals such as education, health, nutrition and access to clean drinking water and improved sanitation. Moreover, gender equity remains a major challenge in most countries. In this paper, we examine the role that foreign aid plays in generating these outcomes, using panel data from OECD-DAC on the sectoral allocation of development aid, in conjunction with country-level data on public expenditures, human development outcomes and other economic, social and political indicators. Specifically, the paper attempts to assess whether the volume of aid and its sectoral allocation have an impact on human development outcomes and gender equity. We find that the impact of aid on many of the outcomes we study is .../
\end{abstract}

Keywords: foreign aid, human development, gender equity, education, health JEL classification: O1, O2, D0, E0

\footnotetext{
Copyright (c) UNU-WIDER 2013

1Hampshire College, USA, Email: lpickbourn@gmail.com; 2University of Massachusetts at Amherst, USA, Email: ndiku@econs.umass.edu

This study has been prepared within the UNU-WIDER project 'ReCom-Research and Communication on Foreign Aid', directed by Tony Addison and Finn Tarp.

UNU-WIDER gratefully acknowledges specific programme contributions from the governments of Denmark (Ministry of Foreign Affairs, Danida) and Sweden (Swedish International Development Cooperation Agency-Sida) for ReCom. UNU-WIDER also gratefully acknowledges core financial support to its work programme from the governments of Denmark, Finland, Sweden, and the United Kingdom.
} 
... largely dependent on initial levels of human development and per capita income. The results on the impact of aid vary by type of development outcome. While aid appears to be effective in reducing maternal mortality as well as the gender gap in youth literacy regardless of initial conditions, its effects are at best mixed for other indicators. The paper points to a number of policy issues that deserve further investigation.

\section{Acknowledgements}

Paper prepared under the Gender Equality theme of the UNU-WIDER ReCom project. The authors thank Malokele Nanivazo for constructive comments on an earlier draft of this paper.

The World Institute for Development Economics Research (WIDER) was established by the United Nations University (UNU) as its first research and training centre and started work in Helsinki, Finland in 1985. The Institute undertakes applied research and policy analysis on structural changes affecting the developing and transitional economies, provides a forum for the advocacy of policies leading to robust, equitable and environmentally sustainable growth, and promotes capacity strengthening and training in the field of economic and social policy making. Work is carried out by staff researchers and visiting scholars in Helsinki and through networks of collaborating scholars and institutions around the world.

www.wider.unu.edu

publications@wider.unu.edu

UNU World Institute for Development Economics Research (UNU-WIDER)

Katajanokanlaituri 6 B, 00160 Helsinki, Finland

Typescript prepared by Janis Vehmaan-Kreula at UNU-WIDER.

The views expressed in this publication are those of the author(s). Publication does not imply endorsement by the Institute or the United Nations University, nor by the programme/project sponsors, of any of the views expressed. 


\section{Introduction}

While developing countries have made progress in human development since the turn of the century, many are still lagging behind in important development goals such as education, health, nutrition and access to clean drinking water and modern sanitation. Moreover, large gender gaps remain along many dimensions, including access to education, health, and other social services. The evidence suggests that growth and development are not gender-neutral. At the same time there is growing evidence that improvement in gender development outcomes have important implications both at the micro and aggregate level. At the micro level, improvement in access to education and health for women has a substantial positive impact on the wellbeing of children. In addition, improvements in health and education for women as well as increased access to productive assets are accompanied by large economywide productivity gains (Agenor et al. 2010; Seguino 2008; World Bank 2011).

Gender equity is still not adequately integrated into policy design, despite the substantial amount of evidence on its importance for economic growth and human development. Efforts to accelerate progress in human development have focused on strategies to mobilize more financial resources both domestically and through increased aid. Yet, there is no consensus as to whether higher aid inflows accelerate growth and development. The empirical evidence suggests that while targeted aid-funded interventions at the micro level tend to produce positive results, the impact of foreign aid at the macro level is harder to document. The evidence reveals a micro-macro paradox regarding the impact of aid on economic development (Clemens et al. 2004; Ndikumana 2012a, 2012b). Attempts to shed light on this paradox have been constrained by the fact that empirical analysis has typically relied on aggregate data to identify linkages between growth and the volume of aid. Yet, in addition to the volume of aid, its allocation across sectors may affect its impact on development outcomes. The allocation of aid may also affect the equity characteristics of economic development including gender equality.

This paper seeks to contribute to the debate on the impact of foreign aid on economic development by exploring the implications of aid allocation by sector for the aiddevelopment nexus. The study focuses on some of the two important dimensions of social and human development namely education and health. It seeks to explore the following questions: does an increase in the volume of foreign aid help ameliorate human development outcomes at the country level? Does the sectoral allocation of official development assistance matter for its impact on human development? Are gender outcomes influenced by the sectoral allocation of foreign aid?

The paper uses aid data from the OECD-DAC that are disaggregated by sector along various dimensions of human development. We investigate the effects of foreign aid on human development and gender equity by considering not only total foreign aid inflows but also aid allocation to social sectors. This analysis may help assess the gains in human development and gender equity from targeting particular social sectors such as education and health in the allocation of foreign aid.

The econometric analysis uses panel data techniques that enable us to control for countryspecific effects using fixed-effects estimations, potential outliers using iterated reweighted 
least squares, and potential endogeneity of the regressors using the generalized method of moments (GMM).

The remainder of the paper is organized as follows. The next section provides a review of the relevant literature on the linkages between foreign aid and human development as well as the importance of gender equity in economic development. Section 3 describes the data and presents the econometric estimation methodology. Section 4 discusses the results and Section 5 concludes.

\section{Literature review}

The on-going debate over the effectiveness of official development assistance has been focused largely on whether or not foreign aid has contributed to enhancing the macroeconomic performance of recipient countries. The evidence on this question is mixed at best. While some studies suggest that aid has been effective in stimulating economic growth (Gormanee et al. 2005a, 2005b; Hansen and Tarp 2000, 2001), others find that the effectiveness of aid is conditional on the policy and institutional environment (Burnside and Dollar 2000; Collier and Dollar 2004). Yet other studies suggest that aid has simply failed to contribute to growth in recipient countries (Boone 1996; Easterly 2006; Rajan and Subramanian 2005).

In contrast, micro-level analyses of aid effectiveness suggest that targeted aid interventions have achieved positive results at the micro level. For example, Mishra and Newhouse (2009) find that aid helps to lower infant mortality in recipient countries, while Michalowa and Weber (2006) and Dreher et al. (2008) find evidence that aid may contribute to increasing primary school enrolment. Gormanee et al. (2005a) also find that aid is associated with improvements in the Human Development Index. As Ndikumana (2012b) notes, the positive results at the micro level may not aggregate into visible positive outcomes at the macro level because of a variety of structural problems in the existing development assistance model. It is this dichotomy between the micro-level and macro-level effectiveness of aid that has contributed to the continuing unease regarding the impact of aid on economic development.

Missing from both the micro- and macro-level analysis of aid effectiveness is an analysis of the impact of aid on gender equity in social and human development outcomes. This is somewhat surprising given the burgeoning literature that points to the links between gender inequality on the one hand and macroeconomic policies and economic growth on the other. A growing number of theoretical and empirical studies suggest that gender inequality in employment outcomes can affect economic growth, although the effect is influenced by the structure of the economy, the macroeconomic policy regime and the extent of gender segregation in employment (Berik et al. 2009; Braunstein 2012). ${ }^{1}$ Gender gaps in education may also constrain economic growth. For example, Klasen and Lamanna (2009) find that gender gaps in education account for between 1.7 per cent to 3.5 per cent of the difference in annual per capita growth rates in East Asia on the one hand, and sub-Saharan Africa (SSA), South Asia and the Middle East on the other. Studies that examine the separate effects of female and male education on growth have found that female education has a stronger positive impact on growth than male education (Kalaitzidakis et al. 2001; Klasen 2002). One possible reason for this is that increasing women's educational attainment has a positive

1 For reviews of this literature, see Stotsky (2006), (Berik and Rodgers 2008), and Braunstein (2008). 
impact on human capital accumulation through its impact on children's health and education outcomes (Xu 2007).

Gender inequality does not merely influence macroeconomic outcomes, it is itself influenced by these outcomes, and in particular, by the macroeconomic policies that are used to achieve these outcomes. An abundance of literature dating back to the 1990s shows that economic policies are not gender-neutral: fiscal and monetary policies can have different impacts on men and women. For example, fiscal austerity resulting in cuts to public spending on health and other subsidies can lead to an intensification of women's unpaid work at home, as women try to compensate for cuts in social services (Deere et al. 1990; Elson and Cagatay 2000; Floro 1995).

In the same vein, public expenditures on investment in social infrastructure that improves access to water and sanitation can reduce women's care burden, allowing them more time to participate in paid employment, which may not only improve their standing within the household, but also has implications for their own well-being and that of their children and for long-term productivity growth (Agenor et al. 2010; Seguino 2008). Programmes designed to expand the social safety net, such as cash transfer programmes, have been found to be particularly successful in improving children's outcomes when they direct resources to women (Adato and Hoddinott 2007; Skoufias and McCafferty 2001).

Clearly, there is mounting global evidence of the substantial economic and social dividends that can be gained from reducing gender inequality. To a greater extent than ever before, the international policy community is paying attention to the subject. The entire 2012 World Development Report by the World Bank is devoted to the issue of gender equity, highlighting its importance both as a development goal in its own right, and as an instrument for development. The report identifies four priority areas for policy: the reduction of female mortality and gender inequality in educational outcomes; improved access to economic opportunities for women; the empowerment of women in the household and society and the reduction of intergenerational gender inequality (World Bank 2011).

To the extent that foreign aid helps to loosen constraints on public expenditure and create the fiscal space for investment in social infrastructure and support for social services such as health care, it has the potential to generate better outcomes for women, with the attendant spill-over effects for the rest of the economy. However, as Richey (2000) notes, aid interventions could potentially impede the achievement of gender equity if the resources provided by these interventions exacerbate existing inequalities between men and women. In this case, this could be seen as another structural deficiency in the development assistance model that would help to explain the micro-macro paradox in aid effectiveness. Given the centrality of gender equity to development practice, and the widespread acknowledgement that the reduction of gender inequality is not only a goal in its own right, but is also a key contributor to sustainable and equitable economic growth, it appears that an analysis of the impact of foreign aid on gender equity and human development is not only timely, but essential. 


\subsection{The data}

In this section, we describe the data used in the empirical analysis and discuss the specification of the econometric models used to explore the impact of aid on human development and gender equity. We also describe the econometric estimation methodology.

We use data on foreign aid compiled by the OECD based on information reported by donors on bilateral official development assistance commitments to the Creditor Reporting System (CRS) database. The database includes information by donor, recipient, sector, and other classifications such as aid modality and type of financing instrument (grants or loans). We collapse aid from all donors to obtain total aid by recipient country and total aid by sector.

The original dataset for foreign aid contains information for 141 recipient countries. But the actual number of countries included in the empirical analysis is smaller due to data availability as indicated in the tables of regression results presented in the next section. Data on social and human development and gender equity are from the UNDP online database. Country-level social and economic indicators used in the empirical analysis are taken from the World Bank's World Development Indicators (online) and governance indicators are from the International Country Risk Guide (ICRG). The data on foreign aid are available from 1973 to 2010. However, the actual length of the time series used in the empirical analysis varies depending on the availability of data on other regression variables, especially the indicators of human development. While the series for maternal mortality and youth literacy are available from 1975, the other indicators of human development start in 1980 or later. The summary statistics for the variables used in the regression analysis are reported in Table A1. The names, definitions of the variables, coverage (time span), and data sources are reported in Table A2.

By using disaggregated data on aid allocation by sector, this study can contribute to the debate on the developmental impact of aid which has primarily relied on evidence at the macroeconomic level. It can thus shed light on the macro-micro paradox of aid effectiveness (Clemens et al. 2004; Ndikumana 2012a, 2012b).

\subsection{Model specification and econometric estimation methodology}

The econometric analysis in this study aims at exploring the linkages between foreign aid on one hand and human development outcomes in general and gender equity in particular on the other hand. We use regression analysis to examine the impact of total foreign aid to a country as well as its sectoral allocation on measures of human development and gender equity. Human development may be measured at various dimensions. In this analysis we focus on health and education. To assess the link between aid and gender equity outcomes, we explore the impact of aid on maternal mortality and gender parity in youth literacy, and on gender inequality. In the presentation of the methodology below, we refer to these dimensions of human development as sectors. The sectors are indexed by $s$, which varies from 1 to $S$.

The impact of foreign aid on human development is investigated by estimating an econometric model where measures of overall human development are regressed directly on total aid at the country level. As an overall measure of human development, we use the 
UNDP's human development index and its variant that accounts for gender equity. The model is specified as follows:

$$
y_{i t}=b_{0}+\alpha A_{i t}+\mathbf{x}_{i t}^{\prime} \boldsymbol{\beta}+\mu_{i}+\varepsilon_{i t}
$$

where for a country $i$ in year $t, y$ is an indicator of human development or gender development, $A$ is total aid to the country, $\mathrm{x}$ is a vector of control variables consisting of determinants of human development other than aid, $\mu$ is a term that accounts for unobserved country-specific factors, and $\varepsilon$ is a random error term. As control variables we use sectoral public expenditure, access to water, access to sanitation, the age dependency ratio, and governance measured by a corruption index and a government stability index. We also include regional dummy variables for SSA, East Asia and Latin America and the Caribbean.

A variant of the above model is used to estimate the impact of aid at the sectoral level on the specific sector-level development outcome. Equation 1 becomes:

$$
y_{s, i t}=b_{0}+\alpha A_{s, i t}+\mathbf{x}_{i t}^{\prime} \boldsymbol{\beta}+\mu_{i}+\varepsilon_{i t}
$$

where $A_{s}$ is aid disbursement targeted to a specific sector $s$ such as education and health or gender-specific measures of education and health outcomes. It may be argued that sectorlevel development is affected by both the volume of aid targeted to that particular sector as well as total aid flows to the country. Equation 2 can be modified to test this conjecture by including a measure of total aid together with the share of aid to the specific sector in total aid. This yields the following equation:

$$
y_{s, i t}=b_{0}+\alpha A_{i t}+\theta a_{s, i t}+\mathbf{x}_{i t}^{\prime} \boldsymbol{\beta}+\mu_{i}+\varepsilon_{i t}
$$

where $a_{s}=A_{s} / A$ is the share of total aid going to sector $s$.

In estimating equations 1-3 we take into account country-specific time-invariant factors using panel data fixed-effects estimation techniques. Given the large size of the sample and imperfections in data reporting, the data may contain a substantial number of outliers that may potentially bias the results. We account for this potential problem by using the iteratively reweighted least squares (IRLS) method. Finally we use the GMM to account for possible endogeneity of regressors. Using this variety of estimation techniques enables us to assess the robustness of the results. The results of the econometric analysis are presented in the next section.

\section{$4 \quad$ Econometric results}

We present two categories of results. The first category consists of regression results for human development both at the aggregate level and at the sector level, namely education and health. The second category includes the results for gender-specific outcomes, namely gender inequality in human development, education (youth literacy) and health (maternal mortality). 


\subsection{Foreign aid and human development}

The overall impact of foreign aid on human development as measured by the Human Development Index (HDI) appears to depend on initial human development and initial per capita income. In the OLS and IRLS regressions in which we control for these two factors, an increase in total foreign aid is associated with a lower level of human development (Columns 1-4, Table 1).

However, when we do not control for initial conditions, higher foreign aid is associated with higher levels of human development (Columns 5-6, Table 1). A possible explanation for this result is that aid is targeted to countries that have low initial levels of human development, which also develop more slowly. The positive and statistically significant coefficients on the initial HDI and GDP per capita variables suggest that countries which start out with a higher level of human development or per capita income continue to out-perform countries with lower initial levels of human development or per capita income. At the same time, the development assistance model is designed in such a way that countries at the bottom of the income/HDI scale receive relatively more aid than other countries. These two facts explain the negative association between the volume of aid and overall human development when initial conditions are accounted for.

Other important determinants of human development include public spending on health, access to water and government stability, all of which are associated with improvements in the HDI. Significant improvements in the HDI appear to have occurred after 2000, as indicated by the positive and statistically significant coefficient on the post-2000 dummy variable. However, South Asia and SSA appear to be lagging behind other regions. Latin America, on the other hand, has seen improvements in human development. The agedependency ratio consistently has a negative and statistically significant effect on human development across all the regressions.

Given the importance of initial conditions in determining the impact of aid on human development, we wondered whether receiving foreign aid leads to convergence or divergence among recipient countries. In other words, do recipient countries with high initial levels of human development or per capita GDP see more rapid gains in human development compared to recipient countries with low initial levels of human development, and what is the impact of foreign aid on the rate of convergence or divergence? We therefore perform a cross-sectional regression of the change in HDI over the sample period on the same set of variables.

We find that the initial HDI is associated with slower rate of change in the HDI, suggesting some degree of convergence among countries. However, increases in aid as a share of GDP are associated with a slower rate of change in the HDI, suggesting that aid does not help the poorest countries to catch up (Table 2). Indeed, observation of the data reveals large gaps in HDI across regions. In particular, SSA continues to lag behind other regions in most dimensions of human development. The econometric results taken together with the observed trends in human development suggest that countries that started at the bottom of the scale have not progressed sufficiently to catch up with their more advanced counterparts, and aid has not helped to hasten the catch-up process. 
Table 1: Impact of aid on overall human development

\begin{tabular}{|c|c|c|c|c|c|c|}
\hline Variables & $\begin{array}{c}(1) \\
\text { OLS } \\
\text { With initial } \\
\text { HDI }\end{array}$ & $\begin{array}{c}\text { (2) } \\
\text { OLS } \\
\text { With initial GDP } \\
\text { per capita }\end{array}$ & $\begin{array}{c}(3) \\
\text { IRLS } \\
\text { With initial } \\
\text { HDI }\end{array}$ & $\begin{array}{c}\text { (4) } \\
\text { IRLS } \\
\text { With initial GDP } \\
\text { per capita }\end{array}$ & $\begin{array}{l}(5) \\
F E\end{array}$ & $\begin{array}{c}(6) \\
\text { GMM }\end{array}$ \\
\hline Total aid / GDP & $\begin{array}{l}-0.0001^{*} \\
(0.061)\end{array}$ & $\begin{array}{c}-0.0002^{\star} \\
(0.062)\end{array}$ & $\begin{array}{c}-0.0003^{\star \star \star} \\
(0.000)\end{array}$ & $\begin{array}{c}-0.0002^{\star \star \star} \\
(0.000)\end{array}$ & $\begin{array}{c}0.00004^{\star \star} \\
(0.029)\end{array}$ & $\begin{array}{c}0.00002^{\star \star \star} \\
(0.000)\end{array}$ \\
\hline $\begin{array}{l}\text { Public expenditure } \\
\text { on health / GDP }\end{array}$ & $\begin{array}{l}-0.001 \\
(0.523)\end{array}$ & $\begin{array}{l}0.003^{\star} \\
(0.066)\end{array}$ & $\begin{array}{l}-0.000 \\
(0.833)\end{array}$ & $\begin{array}{l}0.005^{\star \star \star} \\
(0.000)\end{array}$ & $\begin{array}{l}0.005^{\star \star \star} \\
(0.000)\end{array}$ & $\begin{array}{c}0.0008^{\star \star \star} \\
(0.000)\end{array}$ \\
\hline $\begin{array}{l}\text { Public expenditure } \\
\text { on education / GDP }\end{array}$ & $\begin{array}{c}0.001 \\
(0.457)\end{array}$ & $\begin{array}{c}0.000 \\
(0.817)\end{array}$ & $\begin{array}{c}0.000 \\
(0.679)\end{array}$ & $\begin{array}{l}-0.001 \\
(0.387)\end{array}$ & $\begin{array}{c}0.001 \\
(0.128)\end{array}$ & $\begin{array}{c}-0.0006^{\star \star \star} \\
(0.005)\end{array}$ \\
\hline $\begin{array}{l}\text { Age dependency } \\
\text { ratio }\end{array}$ & $\begin{array}{l}-0.001^{\star \star \star} \\
(0.000)\end{array}$ & $\begin{array}{c}-0.001^{\star \star \star} \\
(0.000)\end{array}$ & $\begin{array}{c}-0.001^{\star * \star} \\
(0.000)\end{array}$ & $\begin{array}{c}-0.002^{\star \star \star} \\
(0.000)\end{array}$ & $\begin{array}{c}-0.002^{\star \star *} \\
(0.000)\end{array}$ & $\begin{array}{c}-0.0002^{\star \star \star} \\
(0.000)\end{array}$ \\
\hline Access to sanitation & $\begin{array}{l}-0.000 \\
(0.647)\end{array}$ & $\begin{array}{l}-0.000 \\
(0.859)\end{array}$ & $\begin{array}{l}-0.000 \\
(0.955)\end{array}$ & $\begin{array}{l}-0.000 \\
(0.101)\end{array}$ & $\begin{array}{l}0.002^{\star \star \star} \\
(0.000)\end{array}$ & $\begin{array}{c}-0.0001^{\star \star \star} \\
(0.000)\end{array}$ \\
\hline Access to water & $\begin{array}{l}0.002^{\star \star \star} \\
(0.000)\end{array}$ & $\begin{array}{l}0.003^{\star \star \star} \\
(0.000)\end{array}$ & $\begin{array}{l}0.002^{\star * *} \\
(0.000)\end{array}$ & $\begin{array}{l}0.003^{\star \star \star} \\
(0.000)\end{array}$ & $\begin{array}{l}0.002^{\star \star \star} \\
(0.000)\end{array}$ & $\begin{array}{c}0.0004^{\star \star \star} \\
(0.000)\end{array}$ \\
\hline $\begin{array}{l}\text { Government } \\
\text { stability }\end{array}$ & $\begin{array}{l}0.003^{\star * *} \\
(0.001)\end{array}$ & $\begin{array}{l}0.003^{\star} \\
(0.081)\end{array}$ & $\begin{array}{l}0.004^{\star \star \star} \\
(0.000)\end{array}$ & $\begin{array}{c}0.001 \\
(0.331)\end{array}$ & $\begin{array}{l}-0.000 \\
(0.984)\end{array}$ & $\begin{array}{c}0.0001^{\star \star \star} \\
(0.000)\end{array}$ \\
\hline Initial HDI & $\begin{array}{l}0.680^{\star \star \star} \\
(0.000)\end{array}$ & & $\begin{array}{l}0.664^{\star \star \star} \\
(0.000)\end{array}$ & & & \\
\hline $\begin{array}{l}\text { Initial per } \\
\text { capita GDP }\end{array}$ & & $\begin{array}{l}0.019 * * * \\
(0.000)\end{array}$ & & $\begin{array}{l}0.024^{\star \star \star} \\
(0.000)\end{array}$ & & \\
\hline post-2000 & $\begin{array}{l}0.026^{\star \star \star} \\
(0.000)\end{array}$ & $\begin{array}{c}0.020 * \star \star \\
(0.001)\end{array}$ & $\begin{array}{l}0.028 * \star \star \\
(0.000)\end{array}$ & $\begin{array}{c}0.020 \star \star \star \\
(0.000)\end{array}$ & & \\
\hline $\begin{array}{l}\text { Latin America \& } \\
\text { Caribbean }\end{array}$ & $\begin{array}{l}-0.004 \\
(0.426)\end{array}$ & $\begin{array}{l}0.032^{\star \star \star} \\
(0.000)\end{array}$ & $\begin{array}{l}-0.003 \\
(0.542)\end{array}$ & $\begin{array}{l}0.019 * * * \\
(0.003)\end{array}$ & & \\
\hline South Asia & $\begin{array}{l}-0.047^{\star \star \star} \\
(0.000)\end{array}$ & $\begin{array}{c}-0.142^{\star \star \star} \\
(0.000)\end{array}$ & $\begin{array}{c}-0.049^{\star \star *} \\
(0.000)\end{array}$ & $\begin{array}{c}-0.145^{\star \star \star} \\
(0.000)\end{array}$ & & \\
\hline Sub-Saharan Africa & $\begin{array}{c}-0.070 * \star \star \\
(0.000)\end{array}$ & $\begin{array}{l}-0.117^{\star \star *} \\
(0.000)\end{array}$ & $\begin{array}{c}-0.067^{\star \star \star} \\
(0.000)\end{array}$ & $\begin{array}{c}-0.118^{\star \star \star} \\
(0.000)\end{array}$ & & \\
\hline Lagged HDI & & & & & & $\begin{array}{l}0.903^{\star \star \star} \\
(0.000)\end{array}$ \\
\hline Constant & $\begin{array}{l}0.150 * \star \star \\
(0.000)\end{array}$ & $\begin{array}{l}0.327^{\star \star \star} \\
(0.000)\end{array}$ & $\begin{array}{l}0.154^{\star \star \star} \\
(0.000)\end{array}$ & $\begin{array}{l}0.325^{\star \star \star} \\
(0.000)\end{array}$ & $\begin{array}{l}0.438^{\star \star \star} \\
(0.000)\end{array}$ & $\begin{array}{l}0.047^{\star \star \star} \\
(0.000)\end{array}$ \\
\hline Overall R-squared & 0.919 & 0.774 & 0.916 & 0.800 & 0.610 & \\
\hline Within R-squared & & & & & 0.740 & \\
\hline Between R-squared & & & & & 0.640 & \\
\hline $\begin{array}{l}\text { Test for 1-st order } \\
\text { autocorrelation }\end{array}$ & & & & & $\begin{array}{l}-4.052 \\
(0.000)\end{array}$ & \\
\hline $\begin{array}{l}\text { Sargan test }\left(\mathrm{H}_{0} \text { : }\right. \\
\text { instruments are valid) }\end{array}$ & & & & & & $\begin{array}{l}65.041 \\
(1.000)\end{array}$ \\
\hline Observations & 906 & 906 & 906 & 906 & 906 & 827 \\
\hline Number of countries & & & & & 71 & 69 \\
\hline
\end{tabular}

Notes: The dependent variable is the human development index. Robust p-values are given in parentheses. The *'s stand for the level of statistical significance: ${ }^{* \star} p<0.01,{ }^{* \star} p<0.05,{ }^{*} p<0.1$.

Source: Authors' estimations. 
Table 2: Impact of aid on the change in HDI (current HDI - initial HDI)

\begin{tabular}{|c|c|c|c|c|}
\hline & (1) & (2) & (3) & (4) \\
\hline Variables & $\begin{array}{c}\text { OLS } \\
\text { With initial HDI }\end{array}$ & $\begin{array}{c}\text { OLS } \\
\text { With initial GDP per } \\
\text { capita }\end{array}$ & $\begin{array}{c}\text { IRLS } \\
\text { With initial HDI }\end{array}$ & $\begin{array}{c}\text { IRLS } \\
\text { With initial GDP per } \\
\text { capita }\end{array}$ \\
\hline \multirow[t]{2}{*}{ Total aid / GDP } & $-0.001^{\star \star \star}$ & $-0.0004^{\star \star}$ & $-0.001^{\star \star \star}$ & $-0.0004^{\star}$ \\
\hline & $(0.001)$ & $(0.029)$ & $(0.001)$ & $(0.076)$ \\
\hline Public expenditure on & 0.001 & -0.003 & -0.003 & -0.005 \\
\hline health / GDP & $(0.835)$ & $(0.527)$ & $(0.398)$ & $(0.317)$ \\
\hline Public expenditure on & -0.003 & -0.002 & -0.003 & -0.001 \\
\hline education / GDP & $(0.289)$ & $(0.698)$ & $(0.362)$ & $(0.798)$ \\
\hline \multirow[t]{2}{*}{ Age dependency ratio } & $-0.001^{*}$ & -0.0001 & $-0.002^{\star \star \star}$ & -0.0003 \\
\hline & $(0.086)$ & $(0.894)$ & $(0.006)$ & $(0.635)$ \\
\hline \multirow[t]{2}{*}{ Access to sanitation } & 0.000 & 0.0001 & 0.0003 & 0.0001 \\
\hline & $(0.881)$ & $(0.880)$ & $(0.340)$ & $(0.835)$ \\
\hline \multirow[t]{2}{*}{ Access to water } & $0.001^{*}$ & 0.001 & 0.001 & 0.001 \\
\hline & $(0.073)$ & $(0.257)$ & $(0.110)$ & $(0.424)$ \\
\hline \multirow[t]{2}{*}{ Government stability } & $0.011^{\star \star}$ & $0.012^{\star \star}$ & $0.014^{\star \star \star}$ & $0.014^{\star \star}$ \\
\hline & $(0.010)$ & $(0.032)$ & $(0.001)$ & $(0.020)$ \\
\hline \multirow[t]{2}{*}{ Initial HDI } & $-0.426 * \star \star$ & & 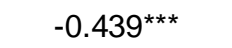 & \\
\hline & $(0.000)$ & & $(0.000)$ & \\
\hline \multirow[t]{2}{*}{ Initial per capita GDP } & & -0.009 & & -0.007 \\
\hline & & $(0.433)$ & & $(0.557)$ \\
\hline Latin America \& & 0.013 & 0.002 & $0.028^{*}$ & 0.003 \\
\hline Caribbean & $(0.521)$ & $(0.943)$ & $(0.097)$ & $(0.905)$ \\
\hline \multirow[t]{2}{*}{ South Asia } & $-0.042^{\star \star}$ & 0.034 & -0.039 & 0.027 \\
\hline & $(0.016)$ & $(0.147)$ & $(0.229)$ & $(0.511)$ \\
\hline \multirow[t]{2}{*}{ Sub-Saharan Africa } & $-0.067 * \star$ & -0.032 & $-0.040^{*}$ & -0.034 \\
\hline & $(0.023)$ & $(0.279)$ & $(0.060)$ & $(0.227)$ \\
\hline \multirow[t]{2}{*}{ Constant } & $0.248^{\star \star \star}$ & 0.039 & $0.253^{\star \star \star}$ & 0.056 \\
\hline & $(0.001)$ & $(0.740)$ & $(0.006)$ & $(0.643)$ \\
\hline Observations & 62 & 62 & 62 & 62 \\
\hline R-squared & 0.646 & 0.365 & 0.682 & 0.372 \\
\hline
\end{tabular}

Notes: The dependent variable is the different between current HDI and initial HDI. Robust p-values are given in parentheses. The *'s stand for the level of statistical significance: ${ }^{* *} p<0.01,{ }^{* *} p<0.05$, * $\mathrm{p}<0.1$.

Source: Authors' estimations.

\section{Foreign aid and health outcomes}

To examine the impact of foreign aid on health outcomes, we use the UNDP health index. In the absence of controls for initial conditions, an increase in the amount of foreign aid to the health sector as a share of GDP is associated with an improvement in the health index (Table 3 , Columns 5 and 6). However, when we control for initial conditions, the aid variable is associated with a lower health index (Table 4, Columns 1 and 2). The results are unchanged when we use alternative measures of aid, such as total aid as a share of GDP. Aid to the health sector as a share of total aid is also associated with a lower health index, regardless of whether we control for initial conditions or not (Table 4). 
As expected, an increase in the share of public spending going to health and an increase in the proportion of the population with access to improved water sources are associated with an improvement in health outcomes. Likewise, a lower risk of corruption is associated with better health outcomes. Again, higher initial levels of human development and per capita income are associated with better health outcomes. In general, countries have experienced an improvement in health outcomes since 2000. However, while Latin America has seen improvements in health outcomes, countries in South Asia and SSA have not.

\section{Foreign aid and education outcomes}

We use the UNDP education index as an indicator of overall education outcomes. Initial conditions clearly matter: in all the regressions, higher initial levels of HDI and per capita GDP are associated with improvements in the education index. We also find that an increase in aid allocated to education as a share of GDP is associated with an improvement in the education index (Table 5, Columns 5 and 6). When we control for initial per capita income, we find a negative relationship between aid to education and educational outcomes. The result is statistically significant when we include initial HDI (Table 7, Columns 1-4).

An increase in aid to education as a share of total aid is associated with improvements in the education index (Table 6, columns 2, 5, 6). Unlike the case of health outcomes, we find no positive effects of increases in budgetary allocation to the education sector. High age dependency is a constraint to educational outcomes as illustrated in the negative and statistically significant coefficients on this variable in all the regressions. Greater government stability is associated with improvements in education outcomes. SSA and South Asian countries have collectively performed relatively worse than their counterparts in other developing regions as illustrated by the negative and significant coefficients on the dummy variables associated with these two regions.

\subsection{Foreign aid and gender inequality}

The impact of foreign aid on gender inequality appears to depend on initial conditions. Total aid reduces gender inequality in the absence of controls for initial conditions (Table 7, Columns 5-6). However, when we control for initial conditions (Table 7, Columns 1-4), the impact of total aid on gender inequality disappears. Public expenditures on health and education are important in lowering gender inequality. Initial conditions also matter: countries with high initial HDI and GDP per capita have lower gender inequality relative to other countries. Although the countries in the sample have experienced a substantial reduction in gender inequality since 2000, Latin America, South Asia and SSA have higher levels of gender inequality. An interesting and perhaps unsurprising result from the GMM regressions is that gender inequality is persistent over time, as illustrated by the positive and significant coefficient on the lagged dependent variable (Column 6, Table 7). This suggests that countries may be caught in a 'gender inequality trap'. 
Table 3: Impact of aid to health sector on health index: results with total aid to the sector

\begin{tabular}{|c|c|c|c|c|c|c|}
\hline \multirow[b]{2}{*}{ Variables } & (1) & (2) & (3) & (4) & (5) & (6) \\
\hline & $\begin{array}{c}\text { OLS } \\
\text { With initial } \\
\text { HDI }\end{array}$ & $\begin{array}{c}\text { OLS } \\
\text { With initial GDP } \\
\text { per capita }\end{array}$ & $\begin{array}{l}\text { IRLS With } \\
\text { initial HDI }\end{array}$ & $\begin{array}{c}\text { IRLS } \\
\text { With initial GDP } \\
\text { per capita }\end{array}$ & FE & GMM \\
\hline \multirow{2}{*}{$\begin{array}{l}\text { Aid to health } \\
\text { sector / GDP }\end{array}$} & 0.0001 & $-0.004^{\star \star \star}$ & -0.001 & $-0.003^{*}$ & $0.005^{\star \star \star}$ & $0.001^{\star \star \star}$ \\
\hline & $(0.932)$ & $(0.005)$ & $(0.487)$ & $(0.062)$ & $(0.000)$ & $(0.000)$ \\
\hline \multirow{2}{*}{$\begin{array}{l}\text { Public health } \\
\text { expenditure / total } \\
\text { expenditure }\end{array}$} & $0.001^{\star * \star}$ & $0.001^{\star \star \star}$ & $0.001^{\star * \star}$ & $0.002^{\star \star *}$ & 0.0001 & $0.0003^{\star \star *}$ \\
\hline & $(0.000)$ & $(0.001)$ & $(0.000)$ & $(0.000)$ & $(0.616)$ & $(0.000)$ \\
\hline \multirow{4}{*}{$\begin{array}{l}\text { Access to } \\
\text { sanitation } \\
\text { Access to water }\end{array}$} & $0.0004^{\star \star \star}$ & $0.0003^{\star \star}$ & $0.001^{\star \star \star}$ & $0.001^{\star \star \star}$ & $0.001^{\star \star \star}$ & $-0.0002^{\star \star \star}$ \\
\hline & $(0.001)$ & $(0.029)$ & $(0.000)$ & $(0.000)$ & $(0.000)$ & $(0.000)$ \\
\hline & 0.0001 & $0.0004^{*}$ & $0.001^{\star \star \star}$ & $0.001^{\star \star \star}$ & $0.002^{\star \star \star}$ & $0.001^{\star \star \star}$ \\
\hline & $(0.620)$ & $(0.090)$ & $(0.000)$ & $(0.000)$ & $(0.000)$ & $(0.000)$ \\
\hline \multirow[t]{2}{*}{ Adult literacy rate } & $-0.001^{\star \star \star}$ & $0.0005^{\star \star \star}$ & $-0.001^{\star \star \star}$ & -0.000 & $0.002^{\star \star \star}$ & $0.0002^{\star \star \star}$ \\
\hline & $(0.000)$ & $(0.001)$ & $(0.000)$ & $(0.163)$ & $(0.000)$ & $(0.000)$ \\
\hline \multirow[t]{2}{*}{ Age dependency ratio } & $-0.001^{\star \star \star}$ & $-0.001^{\star \star \star}$ & $-0.002^{\star \star \star}$ & $-0.002^{\star \star \star}$ & $-0.001^{\star \star \star}$ & $-0.0002^{\star \star \star}$ \\
\hline & $(0.000)$ & $(0.000)$ & $(0.000)$ & $(0.000)$ & $(0.000)$ & $(0.000)$ \\
\hline \multirow{2}{*}{ Corruption index } & 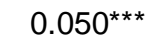 & $0.083^{\star \star \star}$ & 0.008 & $0.029 \star *$ & $0.013^{\star \star}$ & $0.001^{\star \star \star}$ \\
\hline & $(0.005)$ & $(0.000)$ & $(0.505)$ & $(0.039)$ & $(0.023)$ & $(0.000)$ \\
\hline \multirow{4}{*}{$\begin{array}{l}\text { Government } \\
\text { stability } \\
\text { Initial HDI }\end{array}$} & 0.002 & 0.002 & $0.003^{* \star *}$ & 0.002 & $-0.002^{\star \star \star}$ & $-0.0002^{\star \star \star}$ \\
\hline & $(0.130)$ & $(0.169)$ & $(0.004)$ & $(0.158)$ & $(0.000)$ & $(0.000)$ \\
\hline & $0.398^{\star \star \star}$ & & $0.371^{\star \star \star}$ & & & \\
\hline & $(0.000)$ & & $(0.000)$ & & & \\
\hline \multirow[t]{2}{*}{ Initial per capita GDP } & & $0.006^{\star}$ & & $0.006^{\star *}$ & & \\
\hline & & $(0.052)$ & & $(0.017)$ & & \\
\hline \multirow[t]{2}{*}{ post-2000 } & $0.027^{\star \star \star}$ & $0.036^{\star \star \star}$ & $0.021^{\star \star \star}$ & $0.026^{\star \star \star}$ & & \\
\hline & $(0.000)$ & $(0.000)$ & $(0.000)$ & $(0.000)$ & & \\
\hline \multirow{2}{*}{$\begin{array}{l}\text { Latin America \& } \\
\text { Caribbean }\end{array}$} & 0.008 & $0.028^{\star \star \star}$ & 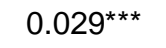 & $0.042^{\star \star \star}$ & & \\
\hline & $(0.132)$ & $(0.000)$ & $(0.000)$ & $(0.000)$ & & \\
\hline \multirow[t]{2}{*}{ South Asia } & $-0.017^{\star \star \star}$ & $-0.032^{\star \star \star}$ & -0.004 & $-0.025^{\star \star \star}$ & & \\
\hline & $(0.006)$ & $(0.000)$ & $(0.608)$ & $(0.006)$ & & \\
\hline Sub-Saharan & $-0.178^{\star \star \star}$ & $-0.205^{\star \star \star}$ & $-0.135^{\star \star \star}$ & $-0.184^{\star \star \star}$ & & \\
\hline Africa & $(0.000)$ & $(0.000)$ & $(0.000)$ & $(0.000)$ & & \\
\hline $\begin{array}{l}\text { Lagged health } \\
\text { index }\end{array}$ & & & & & & $\begin{array}{l}0.901^{* * *} \\
(0.000)\end{array}$ \\
\hline \multirow[t]{2}{*}{ Constant } & $0.643^{\star \star \star}$ & $0.621^{\star \star \star}$ & $0.640^{\star \star \star}$ & $0.658^{\star \star \star}$ & $0.386^{\star \star \star}$ & $0.037^{\star \star \star}$ \\
\hline & $(0.000)$ & $(0.000)$ & $(0.000)$ & $(0.000)$ & $(0.000)$ & $(0.000)$ \\
\hline Overall R-squared & 0.858 & 0.841 & 0.909 & 0.885 & 0.5271 & \\
\hline Within R-squared & & & & & 0.612 & \\
\hline Between R-squared & & & & & 0.5257 & \\
\hline Test for 1-st & & & & & & -1.038 \\
\hline order autocorrelation & & & & & & $(0.030)$ \\
\hline Sargan test $\left(\mathrm{H}_{0}\right.$ : & & & & & & 71.720 \\
\hline instruments are valid) & & & & & & $(0.865)$ \\
\hline Observations & 948 & 1,014 & 948 & 1,014 & 1,014 & 934 \\
\hline Number of countries & & & & & 78 & 77 \\
\hline
\end{tabular}

Notes: The dependent variable is the overall health index. Robust p-values are given in parentheses. The *'s stand for the level of statistical significance: ${ }^{* \star} p<0.01,{ }^{* \star} p<0.05,{ }^{*} p<0.1$.

Source: Authors' estimations. 
Table 4: Impact of aid to health sector on health index: results with total aid and the sector share in total aid

\begin{tabular}{|c|c|c|c|c|c|c|}
\hline Variables & $\begin{array}{c}\text { (1) } \\
\text { OLS } \\
\text { With initial } \\
\text { HDI }\end{array}$ & $\begin{array}{c}(2) \\
\text { OLS } \\
\text { With initial GDP } \\
\text { per capita }\end{array}$ & $\begin{array}{c}\text { (3) } \\
\text { IRLS With } \\
\text { initial HDI }\end{array}$ & $\begin{array}{c}(4) \\
\text { IRLS } \\
\text { With initial GDP } \\
\text { per capita }\end{array}$ & $\begin{array}{l}(5) \\
\mathrm{FE}\end{array}$ & $\begin{array}{c}6) \\
\text { GMM }\end{array}$ \\
\hline Total aid / GDP & $\begin{array}{l}-0.0001 \\
(0.843)\end{array}$ & $\begin{array}{l}-0.000^{\star \star} \\
(0.026)\end{array}$ & $\begin{array}{c}-0.00003 \\
(0.500)\end{array}$ & $\begin{array}{l}-0.0001^{*} \\
(0.075)\end{array}$ & $\begin{array}{c}0.0001^{* \star *} \\
(0.000)\end{array}$ & $\begin{array}{c}0.00001^{* \star \star} \\
(0.000)\end{array}$ \\
\hline $\begin{array}{l}\text { Aid to health / } \\
\text { total aid }\end{array}$ & $\begin{array}{l}-0.064^{*} \\
(0.070)\end{array}$ & $\begin{array}{c}-0.107^{\star * \star} \\
(0.005)\end{array}$ & $\begin{array}{l}-0.053^{*} \\
(0.084)\end{array}$ & $\begin{array}{l}-0.053 \\
(0.120)\end{array}$ & $\begin{array}{c}0.015 \\
(0.209)\end{array}$ & $\begin{array}{c}-0.004^{\star \star *} \\
(0.000)\end{array}$ \\
\hline $\begin{array}{l}\text { Public health } \\
\text { expenditure / total } \\
\text { expenditure }\end{array}$ & $\begin{array}{l}0.001^{\star \star \star} \\
(0.000)\end{array}$ & $\begin{array}{l}0.001^{* \star \star} \\
(0.001)\end{array}$ & $\begin{array}{l}0.001^{* * *} \\
(0.000)\end{array}$ & $\begin{array}{l}0.002^{\star \star \star *} \\
(0.000)\end{array}$ & $\begin{array}{l}0.0001 \\
(0.729)\end{array}$ & $\begin{array}{c}0.0003^{\star * *} \\
(0.000)\end{array}$ \\
\hline $\begin{array}{l}\text { Access to } \\
\text { sanitation }\end{array}$ & $\begin{array}{c}0.0004^{\star \star \star} \\
(0.001)\end{array}$ & $\begin{array}{l}0.0003^{* *} \\
(0.038)\end{array}$ & $\begin{array}{l}0.001^{\star \star *} \\
(0.000)\end{array}$ & $\begin{array}{l}0.001^{* * *} \\
(0.000)\end{array}$ & $\begin{array}{c}0.001^{\star \star \star} \\
(0.000)\end{array}$ & $\begin{array}{c}-0.0002^{\star \star \star} \\
(0.000)\end{array}$ \\
\hline Access to water & $\begin{array}{l}0.0001 \\
(0.646)\end{array}$ & $\begin{array}{l}0.0004^{*} \\
(0.079)\end{array}$ & $\begin{array}{l}0.001^{\star * *} \\
(0.000)\end{array}$ & $\begin{array}{l}0.001^{* \star \star} \\
(0.000)\end{array}$ & $\begin{array}{c}0.002^{\star \star *} \\
(0.000)\end{array}$ & $\begin{array}{c}0.0005^{\star \star \star} \\
(0.000)\end{array}$ \\
\hline $\begin{array}{l}\text { Adult literacy } \\
\text { rate }\end{array}$ & $\begin{array}{c}-0.001^{\star \star *} \\
(0.000)\end{array}$ & $\begin{array}{l}0.001^{\star \star \star} \\
(0.001)\end{array}$ & $\begin{array}{c}-0.001^{\star \star \star} \\
(0.000)\end{array}$ & $\begin{array}{l}-0.0002 \\
(0.173)\end{array}$ & $\begin{array}{c}0.002^{\star * *} \\
(0.000)\end{array}$ & $\begin{array}{c}0.0002^{\star * \star} \\
(0.000)\end{array}$ \\
\hline $\begin{array}{l}\text { Age } \\
\text { dependency ratio }\end{array}$ & $\begin{array}{c}-0.001^{\star \star \star} \\
(0.000)\end{array}$ & $\begin{array}{c}-0.001^{\star \star \star} \\
(0.000)\end{array}$ & $\begin{array}{c}-0.002^{\star \star \star} \\
(0.000)\end{array}$ & $\begin{array}{c}-0.002^{\star \star \star} \\
(0.000)\end{array}$ & $\begin{array}{c}-0.001^{\star \star \star} \\
(0.000)\end{array}$ & $\begin{array}{c}-0.0002^{\star \star \star} \\
(0.000)\end{array}$ \\
\hline $\begin{array}{l}\text { Corruption } \\
\text { index }\end{array}$ & $\begin{array}{l}0.050^{\star \star \star} \\
(0.006)\end{array}$ & $\begin{array}{l}0.079^{\star \star \star} \\
(0.000)\end{array}$ & $\begin{array}{c}0.008 \\
(0.511)\end{array}$ & $\begin{array}{l}0.028^{\star} \\
(0.051)\end{array}$ & $\begin{array}{c}0.016^{\star \star *} \\
(0.007)\end{array}$ & $\begin{array}{c}0.0004^{\star \star *} \\
(0.004)\end{array}$ \\
\hline $\begin{array}{l}\text { Government } \\
\text { stability }\end{array}$ & $\begin{array}{l}0.002^{*} \\
(0.100)\end{array}$ & $\begin{array}{c}0.002 \\
(0.104)\end{array}$ & $\begin{array}{l}0.003^{\star * \star} \\
(0.002)\end{array}$ & $\begin{array}{c}0.002 \\
(0.115)\end{array}$ & $\begin{array}{c}-0.002^{\star \star \star} \\
(0.000)\end{array}$ & $\begin{array}{c}-0.0002^{\star \star \star} \\
(0.000)\end{array}$ \\
\hline Initial HDI & $\begin{array}{l}0.395^{\star \star \star} \\
(0.000)\end{array}$ & & $\begin{array}{l}0.370^{\star \star \star} \\
(0.000)\end{array}$ & & & \\
\hline $\begin{array}{l}\text { Initial per } \\
\text { capita GDP }\end{array}$ & & $\begin{array}{l}0.006^{\star *} \\
(0.037)\end{array}$ & & $\begin{array}{l}0.006^{\star *} \\
(0.016)\end{array}$ & & \\
\hline post-2000 & $\begin{array}{l}0.028^{\star \star \star} \\
(0.000)\end{array}$ & $\begin{array}{l}0.037^{\star \star \star} \\
(0.000)\end{array}$ & $\begin{array}{l}0.021^{\star * \star} \\
(0.000)\end{array}$ & $\begin{array}{l}0.026^{\star \star *} \\
(0.000)\end{array}$ & & \\
\hline Latin America & $0.010^{*}$ & $0.030^{\star \star \star}$ & $0.030^{* \star \star}$ & $0.044^{* \star *}$ & & \\
\hline \& Caribbean & $(0.088)$ & $(0.000)$ & $(0.000)$ & $(0.000)$ & & \\
\hline South Asia & $\begin{array}{l}-0.014^{\star *} \\
(0.021)\end{array}$ & $\begin{array}{l}-0.029^{\star \star \star} \\
(0.000)\end{array}$ & $\begin{array}{l}-0.002 \\
(0.800)\end{array}$ & $\begin{array}{l}-0.023^{\star *} \\
(0.012)\end{array}$ & & \\
\hline $\begin{array}{l}\text { Sub-Saharan } \\
\text { Africa }\end{array}$ & $\begin{array}{c}-0.177^{\star \star \star} \\
(0.000)\end{array}$ & $\begin{array}{c}-0.204^{\star \star \star} \\
(0.000)\end{array}$ & $\begin{array}{c}-0.134^{\star \star \star} \\
(0.000)\end{array}$ & $\begin{array}{c}-0.182^{\star \star \star} \\
(0.000)\end{array}$ & & \\
\hline $\begin{array}{l}\text { Lagged health } \\
\text { index }\end{array}$ & & & & & & $\begin{array}{l}0.910^{\star \star *} \\
(0.000)\end{array}$ \\
\hline Constant & $\begin{array}{l}0.641^{\star * *} \\
(0.000)\end{array}$ & $\begin{array}{l}0.619^{\star \star \star} \\
(0.000)\end{array}$ & $\begin{array}{l}0.637^{\star * *} \\
(0.000)\end{array}$ & $\begin{array}{l}0.656^{\star \star \star} \\
(0.000)\end{array}$ & $\begin{array}{c}0.382^{* * *} \\
(0.000)\end{array}$ & $\begin{array}{l}0.034^{\star * *} \\
(0.000)\end{array}$ \\
\hline Overall R-squared & 0.859 & 0.842 & 0.908 & 0.885 & 0.542 & \\
\hline Within R-Squared & & & & & 0.594 & \\
\hline Between R-squared & & & & & 0.538 & \\
\hline $\begin{array}{l}\text { Test for 1-st order } \\
\text { autocorrelation }\end{array}$ & & & & & & $\begin{array}{l}-.71831 \\
(0.473)\end{array}$ \\
\hline $\begin{array}{l}\text { Sargan test }\left(\mathrm{H}_{0} \text { : }\right. \\
\text { instruments are valid) }\end{array}$ & & & & & & $\begin{array}{l}67.636 \\
(0.928)\end{array}$ \\
\hline Observations & 948 & 1,014 & 948 & 1,014 & 1,014 & 934 \\
\hline Number of countries & & & & & 78 & 77 \\
\hline
\end{tabular}

Notes: The dependent variable is the overall health index. Robust $p$-values are given in parentheses. The *'s stand for the level of statistical significance: ${ }^{\star \star \star} p<0.01,{ }^{* \star} p<0.05,{ }^{*} p<0.1$.

Source: Authors' estimations. 
Table 5: Impact of aid on education index: regressions with total aid to the sector

\begin{tabular}{|c|c|c|c|c|c|c|}
\hline Variables & $\begin{array}{c}(1) \\
\text { OLS } \\
\text { With initial } \\
\text { HDI }\end{array}$ & $\begin{array}{c}\text { (2) } \\
\text { OLS } \\
\text { With initial } \\
\text { GDP per capita }\end{array}$ & $\begin{array}{c}(3) \\
\text { IRLS } \\
\text { With initial } \\
\text { HDI }\end{array}$ & $\begin{array}{c}(4) \\
\text { IRLS } \\
\text { With initial GDP } \\
\text { per capita }\end{array}$ & $\begin{array}{l}\text { (5) } \\
\text { FE }\end{array}$ & $\begin{array}{c}(6) \\
\text { GMM }\end{array}$ \\
\hline $\begin{array}{l}\text { Aid to education } \\
\text { sector / GDP }\end{array}$ & $\begin{array}{l}-0.0002 \\
(0.862)\end{array}$ & $\begin{array}{l}-0.004^{\star \star \star} \\
(0.010)\end{array}$ & $\begin{array}{c}-0.0001 \\
(0.935)\end{array}$ & $\begin{array}{l}-0.004^{*} \\
(0.077)\end{array}$ & $\begin{array}{l}0.005^{\star \star \star} \\
(0.000)\end{array}$ & $\begin{array}{l}0.001^{\star \star \star} \\
(0.000)\end{array}$ \\
\hline $\begin{array}{l}\text { Public } \\
\text { expenditure on } \\
\text { education / GDP }\end{array}$ & $\begin{array}{l}-0.001 \\
(0.329)\end{array}$ & $\begin{array}{c}0.001 \\
(0.500)\end{array}$ & $\begin{array}{l}-0.002 \\
(0.159)\end{array}$ & $\begin{array}{c}0.002 \\
(0.389)\end{array}$ & $\begin{array}{c}0.001 \\
(0.164)\end{array}$ & $\begin{array}{c}-0.0003^{\star \star \star} \\
(0.000)\end{array}$ \\
\hline $\begin{array}{l}\text { Age dependency } \\
\text { ratio }\end{array}$ & $\begin{array}{l}-0.001^{\star \star \star} \\
(0.000)\end{array}$ & $\begin{array}{l}-0.003^{\star \star \star} \\
(0.000)\end{array}$ & $\begin{array}{l}-0.002^{\star \star \star} \\
(0.000)\end{array}$ & $\begin{array}{l}-0.004^{\star \star \star} \\
(0.000)\end{array}$ & $\begin{array}{l}-0.005^{\star \star \star} \\
(0.000)\end{array}$ & $\begin{array}{c}-0.0004^{\star \star \star} \\
(0.000)\end{array}$ \\
\hline $\begin{array}{l}\text { Government } \\
\text { stability }\end{array}$ & $\begin{array}{l}0.006^{\star * *} \\
(0.000)\end{array}$ & $\begin{array}{l}0.009^{\star * *} \\
(0.000)\end{array}$ & $\begin{array}{l}0.006^{\star \star \star} \\
(0.000)\end{array}$ & $\begin{array}{l}0.008^{\star \star *} \\
(0.000)\end{array}$ & $\begin{array}{l}0.006^{\star \star \star} \\
(0.000)\end{array}$ & $\begin{array}{c}0.0004^{\star \star \star} \\
(0.000)\end{array}$ \\
\hline Initial HDI & $\begin{array}{l}1.044^{\star * \star} \\
(0.000)\end{array}$ & & $\begin{array}{l}1.045^{\star \star \star} \\
(0.000)\end{array}$ & & & \\
\hline $\begin{array}{l}\text { Initial per capita } \\
\text { GDP }\end{array}$ & & $\begin{array}{l}0.038^{\star \star \star} \\
(0.000)\end{array}$ & & $\begin{array}{l}0.039 * * * \\
(0.000)\end{array}$ & & \\
\hline post_2000 & $\begin{array}{l}0.053^{\star \star *} \\
(0.000)\end{array}$ & $\begin{array}{l}0.035^{\star \star *} \\
(0.000)\end{array}$ & $\begin{array}{l}0.055^{\star \star *} \\
(0.000)\end{array}$ & $\begin{array}{l}0.033^{\star \star *} \\
(0.000)\end{array}$ & & \\
\hline $\begin{array}{l}\text { Latin America \& } \\
\text { Caribbean }\end{array}$ & $\begin{array}{c}0.005 \\
(0.328)\end{array}$ & $\begin{array}{l}0.067^{\star * \star} \\
(0.000)\end{array}$ & $\begin{array}{c}0.002 \\
(0.733)\end{array}$ & $\begin{array}{c}0.058^{\star \star \star} \\
(0.000)\end{array}$ & & \\
\hline South Asia & $\begin{array}{c}-0.022^{\star \star *} \\
(0.001)\end{array}$ & $\begin{array}{l}-0.166^{\star \star \star} \\
(0.000)\end{array}$ & $\begin{array}{l}-0.021^{\star \star} \\
(0.015)\end{array}$ & $\begin{array}{c}-0.172^{\star \star \star} \\
(0.000)\end{array}$ & & \\
\hline $\begin{array}{l}\text { Sub-Saharan } \\
\text { Africa }\end{array}$ & $\begin{array}{l}0.020 * \star \star \\
(0.001)\end{array}$ & $\begin{array}{c}-0.066^{\star \star \star} \\
(0.000)\end{array}$ & $\begin{array}{l}0.020 * * \star \\
(0.001)\end{array}$ & $\begin{array}{c}-0.057^{\star \star \star} \\
(0.000)\end{array}$ & & \\
\hline $\begin{array}{l}\text { Lagged education } \\
\text { index }\end{array}$ & & & & & & $\begin{array}{l}0.919 \star \star \star \\
(0.000)\end{array}$ \\
\hline Constant & $\begin{array}{l}0.042^{\star \star} \\
(0.023)\end{array}$ & $\begin{array}{l}0.410^{\star \star *} \\
(0.000)\end{array}$ & $\begin{array}{l}0.050 * \star \star \\
(0.006)\end{array}$ & $\begin{array}{l}0.456^{\star \star \star} \\
(0.000)\end{array}$ & $\begin{array}{l}0.826^{\star \star \star} \\
(0.000)\end{array}$ & $\begin{array}{l}0.074^{\star * *} \\
(0.000)\end{array}$ \\
\hline $\begin{array}{l}\text { Overall R- } \\
\text { squared }\end{array}$ & 0.868 & 0.596 & 0.863 & 0.593 & 0.380 & \\
\hline Within R-squared & & & & & 0.752 & \\
\hline $\begin{array}{l}\text { Between R- } \\
\text { squared }\end{array}$ & & & & & 0.408 & \\
\hline $\begin{array}{l}\text { Test for 1-st order } \\
\text { autocorrelation }\end{array}$ & & & & & & $\begin{array}{l}-3.083 \\
(0.002)\end{array}$ \\
\hline $\begin{array}{l}\text { Sargan test }\left(\mathrm{H}_{0} \text { : }\right. \\
\text { instruments are } \\
\text { valid) }\end{array}$ & & & & & & $\begin{array}{l}71.423 \\
(1.000)\end{array}$ \\
\hline Observations & 1,347 & 1,374 & 1,347 & 1,374 & 1,374 & 1,272 \\
\hline $\begin{array}{l}\text { Number of } \\
\text { countries }\end{array}$ & & & & & 77 & 75 \\
\hline
\end{tabular}

Notes: The dependent variable is the education index. Robust $p$-values are given in parentheses. The ${ }^{*}$ 's stand for the level of statistical significance: ${ }^{* \star} p<0.01,{ }^{* \star} p<0.05,{ }^{*} p<0.1$.

Source: Authors' estimations. 
Table 6: Impact of aid on education index: regressions with total aid and sector share

\begin{tabular}{|c|c|c|c|c|c|c|}
\hline \multirow{3}{*}{ Variables } & (1) & (2) & (3) & (4) & (5) & (6) \\
\hline & OLS & OLS & IRLS With & IRLS & FE & GMM \\
\hline & $\begin{array}{c}\text { With initial } \\
\mathrm{HDI}\end{array}$ & $\begin{array}{l}\text { With initial GDP } \\
\text { per capita }\end{array}$ & & $\begin{array}{l}\text { With initial GDP } \\
\text { per capita }\end{array}$ & & \\
\hline \multirow[t]{2}{*}{ Total aid / GDP } & -0.00001 & -0.0001 & -0.00001 & -0.0002 & $0.0002^{\star \star \star}$ & $0.00001^{\star \star \star}$ \\
\hline & $(0.793)$ & $(0.135)$ & $(0.884)$ & $(0.214)$ & $(0.000)$ & $(0.000)$ \\
\hline \multirow[t]{2}{*}{ Aid to education / } & -0.028 & $0.076^{\star \star \star}$ & -0.027 & 0.044 & $0.036^{\star \star \star}$ & $0.005^{\star \star \star}$ \\
\hline & $(0.121)$ & $(0.003)$ & $(0.122)$ & (0.135) & $(0.000)$ & $(0.000)$ \\
\hline \multicolumn{7}{|l|}{ Total aid } \\
\hline \multirow{2}{*}{$\begin{array}{l}\text { Public expenditure on } \\
\text { education / GDP }\end{array}$} & -0.001 & 0.001 & -0.001 & 0.001 & 0.000 & $-0.0003^{\star \star *}$ \\
\hline & $(0.364)$ & $(0.664)$ & $(0.211)$ & $(0.522)$ & $(0.660)$ & $(0.000)$ \\
\hline Age dependency & $-0.002^{\star \star \star}$ & $-0.003^{\star \star \star}$ & $-0.002^{\star \star \star}$ & $-0.004^{\star \star \star}$ & $-0.005^{\star \star \star}$ & $-0.0004^{\star \star \star}$ \\
\hline ratio & $(0.000)$ & $(0.000)$ & $(0.000)$ & $(0.000)$ & $(0.000)$ & $(0.000)$ \\
\hline Government & $0.006^{\star \star \star}$ & $0.007^{\star \star *}$ & $0.006^{\star \star \star}$ & $0.006^{\star \star *}$ & $0.005^{\star \star *}$ & $0.0003^{\star \star \star}$ \\
\hline stability & $(0.000)$ & $(0.000)$ & $(0.000)$ & $(0.000)$ & $(0.000)$ & $(0.000)$ \\
\hline Initial HDI & $1.044^{\star \star \star}$ & & $1.041^{\star \star \star}$ & & & \\
\hline Initial per capita & $(0.000)$ & $0.035^{\star \star \star}$ & $(0.000)$ & $0.037^{\star \star \star}$ & & \\
\hline GDP & & $(0.000)$ & & $(0.000)$ & & \\
\hline \multirow[t]{2}{*}{ post-2000 } & $0.053^{\star \star \star}$ & $0.029^{\star \star \star}$ & $0.055^{\star \star \star}$ & $0.028^{\star \star \star}$ & & \\
\hline & $(0.000)$ & $(0.000)$ & $(0.000)$ & $(0.000)$ & & \\
\hline Latin America \& & 0.004 & $0.068^{\star \star \star}$ & 0.002 & $0.058^{\star \star \star}$ & & \\
\hline Caribbean & $(0.384)$ & $(0.000)$ & $(0.731)$ & $(0.000)$ & & \\
\hline \multirow[t]{2}{*}{ South Asia } & $-0.026^{\star \star \star}$ & 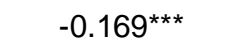 & $-0.025^{\star \star \star}$ & $-0.177^{\star \star \star}$ & & \\
\hline & $(0.000)$ & $(0.000)$ & $(0.006)$ & $(0.000)$ & & \\
\hline Sub-Saharan & $0.021^{\star \star \star}$ & $-0.063^{\star \star \star}$ & $0.022^{\star \star \star}$ & $-0.055^{\star \star \star}$ & & \\
\hline Africa & $(0.001)$ & $(0.000)$ & $(0.000)$ & $(0.000)$ & & \\
\hline Lagged education & & & & & & $0.918^{\star \star \star}$ \\
\hline index & & & & & & $(0.000)$ \\
\hline \multirow[t]{2}{*}{ Constant } & $0.051^{\star \star \star}$ & $0.438^{\star \star \star}$ & $0.063^{\star \star \star}$ & $0.478^{\star \star \star}$ & $0.827^{\star \star *}$ & $0.073^{\star \star \star}$ \\
\hline & $(0.008)$ & $(0.000)$ & $(0.001)$ & $(0.000)$ & $(0.000)$ & $(0.000)$ \\
\hline Overall R-squared & 0.863 & 0.585 & 0.857 & 0.581 & 0.376 & \\
\hline Within R-squared & & & & & 0.747 & \\
\hline Between R-squared & & & & & 0.418 & \\
\hline \multirow{2}{*}{$\begin{array}{l}\text { Test for 1-st order } \\
\text { autocorrelation }\end{array}$} & & & & & -3.070 & \\
\hline & & & & & $(0.002)$ & \\
\hline \multirow{2}{*}{$\begin{array}{l}\text { Sargan test }\left(\mathrm{H}_{0}:\right. \\
\text { instruments are valid) }\end{array}$} & & & & & 68.587 & \\
\hline & & & & & $(1.000)$ & \\
\hline Observations & 1,305 & 1,330 & 1,305 & 1,330 & 1,330 & 1,229 \\
\hline Number of countries & & & & & 77 & 75 \\
\hline
\end{tabular}

Notes: The dependent variable is the education index. Robust $p$-values are given in parentheses. The *'s stand for the level of statistical significance: ${ }^{* \star} p<0.01,{ }^{* *} p<0.05,{ }^{*} p<0.1$.

Source: Authors' estimations. 
Table 7: Impact of aid on gender inequality index

\begin{tabular}{|c|c|c|c|c|c|c|}
\hline & (1) & (2) & (3) & (4) & (5) & (6) \\
\hline \multirow[t]{2}{*}{ Variables } & OLS & OLS With & IRLS & IRLS & FE & GMM \\
\hline & $\begin{array}{l}\text { With initial } \\
\text { HDI }\end{array}$ & $\begin{array}{l}\text { initial GDP per } \\
\text { capita }\end{array}$ & $\begin{array}{l}\text { With initial } \\
\text { HDI }\end{array}$ & $\begin{array}{l}\text { With initial GDP } \\
\text { per capita }\end{array}$ & & \\
\hline \multirow[t]{2}{*}{ Total aid / GDP } & -0.00004 & -0.00004 & -0.0001 & -0.0001 & $-0.0001^{\star}$ & - \\
\hline & $(0.335)$ & $(0.352)$ & $(0.334)$ & $(0.328)$ & $(0.059)$ & $(0.037)$ \\
\hline \multirow[t]{2}{*}{ Public expenditure on } & $-0.004^{\star \star \star}$ & $-0.010 * * *$ & $-0.005^{\star \star \star}$ & $-0.013^{\star * *}$ & - & $0.0001^{* * *}$ \\
\hline & & & & & $0.005^{\star \star \star}$ & \\
\hline health / GDP & $(0.001)$ & $(0.000)$ & $(0.000)$ & $(0.000)$ & $(0.000)$ & $(0.000)$ \\
\hline \multirow{2}{*}{$\begin{array}{l}\text { Public expenditure on } \\
\text { education / GDP }\end{array}$} & -0.001 & -0.002 & $-0.005^{\star \star \star}$ & -0.003 & $-0.005^{\star \star}$ & $0.001^{* * *}$ \\
\hline & $(0.442)$ & $(0.227)$ & $(0.000)$ & $(0.140)$ & $(0.011)$ & $(0.000)$ \\
\hline \multirow[t]{2}{*}{ Age dependency ratio } & $0.002^{\star \star \star}$ & $0.003^{\star \star \star}$ & $0.001^{\star \star \star}$ & $0.002^{\star \star \star}$ & $0.002^{\star \star \star}$ & $0.001^{* \star \star}$ \\
\hline & $(0.000)$ & $(0.000)$ & $(0.000)$ & $(0.000)$ & $(0.000)$ & $(0.000)$ \\
\hline \multirow[t]{3}{*}{ Access to sanitation } & 0.0001 & 0.0001 & $-0.0002^{\star \star}$ & 0.0001 & - & $0.0004^{\star \star *}$ \\
\hline & & & & & $0.004^{\star \star \star}$ & \\
\hline & $(0.308)$ & $(0.709)$ & $(0.044)$ & $(0.230)$ & $(0.000)$ & $(0.000)$ \\
\hline \multirow[t]{2}{*}{ Access to water } & $0.001^{\star *}$ & $-0.001^{\star * \star}$ & 0.000 & $-0.002^{\star \star \star}$ & 0.0003 & $-0.001^{\star * \star}$ \\
\hline & $(0.010)$ & $(0.000)$ & $(0.300)$ & $(0.000)$ & $(0.326)$ & $(0.000)$ \\
\hline \multirow[t]{2}{*}{ Government stability } & $-0.006^{\star \star \star}$ & $-0.006^{\star \star \star}$ & -0.002 & -0.002 & 0.001 & $-0.0002^{\star \star \star}$ \\
\hline & $(0.000)$ & $(0.004)$ & $(0.193)$ & $(0.239)$ & $(0.331)$ & $(0.000)$ \\
\hline \multirow[t]{2}{*}{ Initial HDI } & $-0.582^{\star \star \star}$ & & $-0.642^{\star \star \star}$ & & & \\
\hline & $(0.000)$ & & $(0.000)$ & & & \\
\hline \multirow[t]{2}{*}{ post-2000 } & $-0.023^{\star \star \star}$ & -0.008 & $-0.030 * \star \star$ & -0.006 & & \\
\hline & $(0.000)$ & $(0.280)$ & $(0.000)$ & $(0.326)$ & & \\
\hline Latin America \& & $0.066^{\star \star \star}$ & $0.034^{\star \star \star}$ & $0.067^{\star \star \star}$ & $0.060 * \star \star$ & & \\
\hline Caribbean & $(0.000)$ & $(0.001)$ & $(0.000)$ & $(0.000)$ & & \\
\hline \multirow[t]{2}{*}{ South Asia } & $0.105^{\star \star \star}$ & $0.184^{\star \star \star}$ & 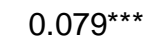 & $0.193^{\star \star \star}$ & & \\
\hline & $(0.000)$ & $(0.000)$ & $(0.000)$ & $(0.000)$ & & \\
\hline \multirow[t]{2}{*}{ Sub-Saharan Africa } & $0.084^{\star \star \star}$ & $0.116^{\star \star \star}$ & $0.080^{\star \star *}$ & $0.131^{\star \star \star}$ & & \\
\hline & $(0.000)$ & $(0.000)$ & $(0.000)$ & $(0.000)$ & & \\
\hline \multirow[t]{2}{*}{ Initial per capita GDP } & & 0.005 & & $-0.009 * \star$ & & \\
\hline & & $(0.318)$ & & $(0.013)$ & & \\
\hline \multirow{2}{*}{$\begin{array}{l}\text { Lagged gender inequality } \\
\text { index }\end{array}$} & & & & & & $0.804^{\star \star \star}$ \\
\hline & & & & & & $(0.000)$ \\
\hline \multirow[t]{2}{*}{ Constant } & $0.612^{\star \star \star}$ & $0.459 * \star \star$ & $0.790 * \star \star$ & $0.542^{\star \star \star}$ & 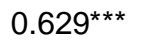 & $0.072^{\star \star \star}$ \\
\hline & $(0.000)$ & $(0.000)$ & $(0.000)$ & $(0.000)$ & $(0.000)$ & $(0.000)$ \\
\hline Overall R-squared & 0.753 & 0.611 & 0.812 & 0.691 & 0.375 & \\
\hline Within R-squared & & & & & 0.444 & \\
\hline Between R-squared & & & & & 0.432 & \\
\hline Test for 1-st order & & & & & & 2.7201 \\
\hline autocorrelation & & & & & & $(0.007)$ \\
\hline $\begin{array}{l}\text { Sargan test }\left(\mathrm{H}_{0} \text { : }\right. \\
\text { instruments are valid })\end{array}$ & & & & & & 43.1393 \\
\hline Observations & 832 & 852 & 832 & 852 & 852 & 739 \\
\hline Number of countries & & & & & 64 & 61 \\
\hline
\end{tabular}

Notes: The dependent variable is the Gender Inequality Index reported by the UNDP. Robust pvalues are given in parentheses. The *'s stand for the level of statistical significance: ${ }^{\star \star \star} p<0.01,{ }^{\star *}$ $p<0.05,{ }^{*} p<0.1$.

Source: Authors' estimations. 


\section{Foreign aid, gender and health outcomes}

To assess the impact of aid on women's health outcomes, we analyse the impact of official development assistance on maternal mortality, since pregnancy-related complications are a leading cause of death among women in many countries. An increase in the total amount of aid going to the health sector as a share of GDP is associated with a reduction in maternal mortality rates in all the regressions (Table 8). Increases in the share of public expenditure on health seem to reduce maternal mortality: the coefficient on this variable is negative and statistically significant in all the regressions except for the IRLS including initial HDI and in fixed-effects estimates (Table 8, columns 3 and 5).

As might be expected, maternal mortality rates decline as access to improved sanitation and water supply improves. Note that the GMM regressions yield statistically significant coefficients but with the wrong sign on these two variables. This apparent inconsistency can be explained by the fact that GMM regressions do not include controls for initial conditions, which as we have seen from the OLS and IRLS results, are important determinants of the maternal mortality rate. In contrast, the fixed effects regressions will capture the effects of the initial conditions, even though they are not directly controlled for in these regressions.

Increases in the age dependency ratio are associated with increases in the maternal mortality rate in all the regressions except for fixed-effects estimates. SSA, Latin America, and South Asia have consistently higher maternal mortality rates than other regions. Note that the coefficients on initial GDP per capita, initial HDI and the post-2000 dummy have opposite (positive) signs than expected.

\section{Foreign aid and gender inequality in education outcomes}

Finally, we examine the impact of foreign development assistance on the gender gap in education outcomes, using the female-male youth literacy ratio as our measure of gender inequality in this dimension. As the results presented in Table 9 indicate, an increase in aid to the education sector as a share of GDP is unambiguously associated with improvements in gender parity in youth literacy. Greater public spending on education also appears to reduce the gender gap in youth literacy, while a higher age-dependency ratio increases it.

Again, initial conditions matter: countries with high initial levels of human development and high initial levels of GDP have greater gender parity in youth literacy outcomes. The gap has been reduced in recent years since 2000, although South Asia continues to lag behind other regions. In contrast, Latin America and SSA have made relatively faster progress towards narrowing the gender gap in youth literacy.

\section{Conclusion}

This paper contributes to the literature on the impact of foreign aid on economic development in two important ways. First the study undertakes an analysis at a disaggregated level and asks the question of whether the allocation of aid across sectors has implications for human development. Second, it explores the impact of the level and sectoral allocation of foreign aid on gender-specific development outcomes, an issue that has received limited attention in the vast literature on the aid-development nexus. 
Table 8: Impact of aid to health sector on maternal mortality rate

\begin{tabular}{|c|c|c|c|c|c|c|}
\hline & (1) & (2) & (3) & (4) & (5) & (6) \\
\hline \multirow[t]{2}{*}{ Variables } & OLS & OLS: & IRLS: & IRLS: & FE & GMM \\
\hline & $\begin{array}{l}\text { With initial } \\
\text { HDI }\end{array}$ & $\begin{array}{l}\text { With initial } \\
\text { income }\end{array}$ & $\begin{array}{l}\text { With initial } \\
\text { HDI }\end{array}$ & $\begin{array}{l}\text { With initial } \\
\text { income }\end{array}$ & & \\
\hline Aid to health & $-11.152^{\star \star \star}$ & $-15.048^{\star \star \star}$ & $-3.934^{\star \star \star}$ & $-8.794^{\star \star \star}$ & $-13.083^{\star \star \star}$ & - \\
\hline sector / GDP & $(0.006)$ & $(0.000)$ & $(0.007)$ & $(0.000)$ & $(0.000)$ & $\begin{array}{l}1.348^{\star \star \star \star} \\
(0.000)\end{array}$ \\
\hline Public health expenditure & -2.643 & $-3.538^{*}$ & -0.454 & $-1.282^{*}$ & -2.013 & $0.072^{\star \star *}$ \\
\hline \multirow{2}{*}{$\begin{array}{l}\text { total expenditure } \\
\text { Access to }\end{array}$} & $(0.186)$ & $(0.073)$ & $(0.379)$ & $(0.075)$ & $(0.129)$ & $(0.000)$ \\
\hline & $-3.063^{\star \star \star}$ & $-3.373^{\star \star \star}$ & $-3.016^{\star \star \star}$ & $-3.229 * \star \star$ & $-12.817^{\star \star \star}$ & $0.566^{\star \star *}$ \\
\hline sanitation & $(0.000)$ & $(0.000)$ & $(0.000)$ & $(0.000)$ & $(0.000)$ & $(0.000)$ \\
\hline \multirow[t]{2}{*}{ Access to water } & $-4.185^{\star \star \star}$ & $-4.423^{\star \star \star}$ & $-1.903^{\star * *}$ & $-2.384^{\star * *}$ & 1.838 & $0.205^{\star \star \star}$ \\
\hline & $(0.000)$ & $(0.000)$ & $(0.000)$ & $(0.000)$ & $(0.216)$ & $(0.002)$ \\
\hline \multirow[t]{2}{*}{ Adult literacy rate } & $-2.050 \star \star$ & -0.522 & $-3.332^{\star \star *}$ & $-1.811^{\star \star *}$ & $-2.593^{\star}$ & $-0.090^{\star}$ \\
\hline & $(0.010)$ & $(0.392)$ & $(0.000)$ & $(0.000)$ & $(0.053)$ & $(0.068)$ \\
\hline \multirow{2}{*}{$\begin{array}{l}\text { Age } \\
\text { dependency ratio }\end{array}$} & $4.038^{\star \star \star}$ & 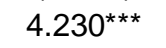 & $1.803^{\star \star \star}$ & $1.981^{\star \star \star}$ & $-6.298 * \star \star$ & $0.396^{\star \star *}$ \\
\hline & $(0.000)$ & $(0.000)$ & $(0.000)$ & $(0.000)$ & $(0.000)$ & $(0.000)$ \\
\hline Corruption & $-157.255^{\star \star \star}$ & -63.366 & $-63.609 * \star \star$ & -19.553 & -45.257 & $\stackrel{-}{-}^{-} 852^{\star \star \star}$ \\
\hline index & $(0.002)$ & $(0.216)$ & $(0.000)$ & $(0.410)$ & $(0.145)$ & $(0.000)$ \\
\hline Government & 0.641 & 2.072 & -0.149 & -0.758 & -2.349 & $\frac{-}{0.539 * \star \star}$ \\
\hline \multirow{3}{*}{$\begin{array}{l}\text { stability } \\
\text { Initial HDI }\end{array}$} & $(0.873)$ & $(0.624)$ & $(0.916)$ & $(0.701)$ & $(0.280)$ & $(0.000)$ \\
\hline & $312.352^{\star \star \star}$ & & $362.017^{* * *}$ & & & \\
\hline & $(0.001)$ & & $(0.000)$ & & & \\
\hline \multirow[t]{2}{*}{ Initial GDP per capita } & & 6.584 & & $11.964^{\star \star \star}$ & & \\
\hline & & $(0.583)$ & & $(0.007)$ & & \\
\hline \multirow[t]{2}{*}{ post-2000 } & 17.715 & $42.746^{\star \star \star}$ & -10.646 & -3.616 & & \\
\hline & $(0.220)$ & $(0.007)$ & $(0.125)$ & $(0.702)$ & & \\
\hline \multirow{2}{*}{$\begin{array}{l}\text { Latin America \& } \\
\text { Caribbean }\end{array}$} & 15.139 & $49.742^{\star \star \star}$ & -0.845 & $16.456^{\star \star}$ & & \\
\hline & $(0.157)$ & $(0.000)$ & $(0.882)$ & $(0.039)$ & & \\
\hline \multirow[t]{2}{*}{ South Asia } & $64.973^{\star \star *}$ & $53.386^{\star \star *}$ & $55.144^{\star \star \star}$ & $38.265^{\star \star \star}$ & & \\
\hline & $(0.000)$ & $(0.005)$ & $(0.000)$ & $(0.005)$ & & \\
\hline Sub-Saharan & $113.611^{\star \star \star}$ & $82.800^{* \star *}$ & $78.845^{\star \star \star}$ & $56.005^{\star \star \star}$ & & \\
\hline Africa & $(0.000)$ & $(0.000)$ & $(0.000)$ & $(0.000)$ & & \\
\hline Lagged maternal mortality & & & & & & $\begin{array}{l}0.984^{\star \star *} \\
(0.000)\end{array}$ \\
\hline \multirow[t]{2}{*}{ Constant } & $556.901^{\star \star \star}$ & $502.917^{\star \star \star}$ & $534.625^{\star \star \star}$ & $545.894^{\star \star \star}$ & 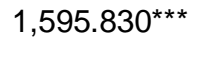 & $\begin{array}{c}- \\
62.87^{\star \star \star}\end{array}$ \\
\hline & $(0.000)$ & $(0.000)$ & $(0.000)$ & $(0.000)$ & $(0.000)$ & $(0.000)$ \\
\hline Overall R-squared & 0.766 & 0.747 & 0.944 & 0.899 & 0.507 & \\
\hline Within R-Squared & & & & & 0.148 & \\
\hline Between R-Squared & & & & & 0.476 & \\
\hline Test for 1-st order & & & & & & -1.221 \\
\hline autocorrelation & & & & & & $(0.222)$ \\
\hline $\begin{array}{l}\text { Sargan test }\left(\mathrm{H}_{0}:\right. \\
\text { instruments are valid })\end{array}$ & & & & & & $\begin{array}{l}63.143 \\
(1.000)\end{array}$ \\
\hline Observations & 506 & 549 & 506 & 549 & 549 & 428 \\
\hline Number of countries & & & & & 75 & 65 \\
\hline
\end{tabular}

Notes: The dependent variable is the maternal mortality rate. Robust p-values are given in parentheses. The *'s stand for the level of statistical significance: ${ }^{* *} p<0.01,{ }^{* *} p<0.05,{ }^{*} p<0.1$.

Source: Authors' estimations. 
Table 9: Impact of aid on female/male youth literacy

\begin{tabular}{|c|c|c|c|c|c|c|}
\hline Variables & $\begin{array}{c}(1) \\
\text { OLS } \\
\text { With initial } \\
\text { HDI }\end{array}$ & $\begin{array}{c}\text { (2) } \\
\text { OLS } \\
\text { With initial } \\
\text { GDP per } \\
\text { capita }\end{array}$ & $\begin{array}{c}(3) \\
\text { IRLS } \\
\text { With initial } \\
\text { HDI }\end{array}$ & $\begin{array}{c}\text { (4) } \\
\text { IRLS } \\
\text { With initial } \\
\text { GDP per } \\
\text { capita }\end{array}$ & $\begin{array}{l}\text { (5) } \\
\mathrm{FE}\end{array}$ & $\begin{array}{c}(6) \\
\text { GMM }\end{array}$ \\
\hline $\begin{array}{l}\text { Aid to education } \\
\text { sector / GDP }\end{array}$ & $\begin{array}{c}0.447^{\star \star \star} \\
(0.002)\end{array}$ & $\begin{array}{l}0.501^{\star \star \star} \\
(0.002)\end{array}$ & $\begin{array}{l}0.395^{\star \star \star} \\
(0.005)\end{array}$ & $\begin{array}{l}0.434^{\star \star \star} \\
(0.007)\end{array}$ & $\begin{array}{l}0.440^{\star \star \star} \\
(0.001)\end{array}$ & $(0.000)$ \\
\hline $\begin{array}{l}\text { Public expenditure } \\
\text { on education / GDP }\end{array}$ & $\begin{array}{l}-0.178 \\
(0.363)\end{array}$ & $\begin{array}{c}-0.318 \\
(0.123 \\
)\end{array}$ & $\begin{array}{c}0.374^{\star \star \star} \\
(0.003)\end{array}$ & $\begin{array}{l}0.266^{*} \\
(0.054)\end{array}$ & $\begin{array}{l}-0.039 \\
(0.820)\end{array}$ & $\begin{array}{l}0.042^{\star \star \star} \\
(0.000)\end{array}$ \\
\hline $\begin{array}{l}\text { Age dependency } \\
\text { ratio }\end{array}$ & $\begin{array}{l}-0.279^{\star \star \star} \\
(0.000)\end{array}$ & $\begin{array}{l}-0.307^{* \star *} \\
(0.000)\end{array}$ & $\begin{array}{l}-0.224^{\star \star \star} \\
(0.000)\end{array}$ & $\begin{array}{l}-0.255^{\star \star \star} \\
(0.000)\end{array}$ & $\begin{array}{l}-0.268^{\star \star \star} \\
(0.000)\end{array}$ & $\begin{array}{c}0.002^{\star \star \star} \\
(0.000)\end{array}$ \\
\hline Government stability & $\begin{array}{l}0.185 \\
(0.188)\end{array}$ & $\begin{array}{l}0.182 \\
(0.267)\end{array}$ & $\begin{array}{l}0.136 \\
(0.201)\end{array}$ & $\begin{array}{l}0.101 \\
(0.401)\end{array}$ & $\begin{array}{l}0.332^{\star * \star} \\
(0.000)\end{array}$ & $\begin{array}{c}- \\
0.003^{\star \star \star} \\
(0.000)\end{array}$ \\
\hline Initial HDI & $\begin{array}{c}33.766^{\star \star \star} \\
(0.000)\end{array}$ & & $\begin{array}{c}10.535^{\star \star \star} \\
(0.000)\end{array}$ & & & \\
\hline Initial per capita GDP & & $\begin{array}{l}3.988^{\star \star \star} \\
(0.000)\end{array}$ & & $\begin{array}{l}1.836^{\star \star *} \\
(0.000)\end{array}$ & & \\
\hline post-2000 & $\begin{array}{l}-1.241^{\star *} \\
(0.036)\end{array}$ & $\begin{array}{c}0.796 \\
(0.243)\end{array}$ & $\begin{array}{l}-1.867^{\star \star \star} \\
(0.000)\end{array}$ & $\begin{array}{l}-1.195^{\star *} \\
(0.024)\end{array}$ & & \\
\hline $\begin{array}{l}\text { Latin America \& } \\
\text { Caribbean }\end{array}$ & $\begin{array}{c}3.498^{\star \star \star} \\
(0.000)\end{array}$ & $\begin{array}{l}5.247^{\star \star \star} \\
(0.000)\end{array}$ & $\begin{array}{l}4.242^{\star \star \star} \\
(0.000)\end{array}$ & $\begin{array}{c}5.111^{\star \star \star} \\
(0.000)\end{array}$ & & \\
\hline South Asia & $\begin{array}{l}-6.587^{* \star *} \\
(0.000)\end{array}$ & $\begin{array}{l}-6.263^{\star \star \star} \\
(0.000)\end{array}$ & $\begin{array}{c}-15.946 * \star \star \\
(0.000)\end{array}$ & $\begin{array}{c}-11.744^{* \star *} \\
(0.000)\end{array}$ & & \\
\hline Sub-Saharan Africa & $\begin{array}{l}2.392^{\star \star *} \\
(0.006)\end{array}$ & $\begin{array}{l}-1.685 \\
(0.106)\end{array}$ & $\begin{array}{l}2.831^{\star \star \star} \\
(0.000)\end{array}$ & $\begin{array}{l}1.470^{\star *} \\
(0.033)\end{array}$ & & \\
\hline $\begin{array}{l}\text { Lagged youth } \\
\text { female/male literacy } \\
\text { ratio }\end{array}$ & & & & & & $\begin{array}{c}0.985^{\star \star \star} \\
(0.000)\end{array}$ \\
\hline Constant & $\begin{array}{l}95.877^{\star \star \star} \\
(0.000)\end{array}$ & $\begin{array}{l}85.611^{\star \star \star} \\
(0.000)\end{array}$ & $\begin{array}{l}103.389 * \star * \\
(0.000)\end{array}$ & $\begin{array}{l}97.491^{\star \star \star} \\
(0.000)\end{array}$ & $\begin{array}{c}108.272^{\star \star \star} \\
(0.000)\end{array}$ & $\begin{array}{l}1.441^{\star \star \star} \\
(0.000)\end{array}$ \\
\hline $\begin{array}{l}\text { Overall R-squared } \\
\text { Within R-squared } \\
\text { Between R-squared }\end{array}$ & 0.484 & 0.501 & 0.492 & 0.444 & $\begin{array}{l}0.3132 \\
0.220 \\
0.3058\end{array}$ & \\
\hline $\begin{array}{l}\text { Test for 1-st order } \\
\text { autocorrelation }\end{array}$ & & & & & & $\begin{array}{l}-1.415 \\
(0.157)\end{array}$ \\
\hline $\begin{array}{l}\text { Sargan test }\left(\mathrm{H}_{0} \text { : }\right. \\
\text { instruments are } \\
\text { valid) }\end{array}$ & & & & & & $\begin{array}{l}65.338 \\
(1.000)\end{array}$ \\
\hline $\begin{array}{l}\text { Observations } \\
\text { Number of countries }\end{array}$ & 1,054 & 1,147 & 1,054 & 1,147 & $\begin{array}{c}1,147 \\
76\end{array}$ & $\begin{array}{c}1,033 \\
71\end{array}$ \\
\hline
\end{tabular}

Notes: The dependent variable is the youth female/male literacy ratio. Robust $p$-values are given in parentheses. The *'s stand for the level of statistical significance: ${ }^{* \star} p<0.01,{ }^{* \star} p<0.05,{ }^{*} p<0.1$.

Source: Authors' estimations. 
Key results emerge from the empirical analysis. We find that the overall impact of aid on human development depends significantly on initial conditions. Specifically, high initial human development is associated with higher human development in subsequent years, and conditional on initial human development, foreign aid is negatively related to subsequent human development. However, when initial conditions are not controlled for, higher aid is associated with higher human development. These results are consistent with the fact that while the poorest countries receive relatively more aid as a result of the design of the development assistance model, they perform relatively poorly compared to higher-income countries because of structural factors that constrain growth and development in poor countries. The results are consistent with the observed persistence of gaps in human development across income levels and regions.

This finding suggests that aid evaluations that do not take into account initial conditions are likely to underestimate the impact of aid on development outcomes. Rather than measuring absolute progress towards the achievement of outcomes, such evaluations should be countryspecific, and should measure progress relative to the starting point of each individual country.

The novelty of this study is in the empirical results with disaggregated data at the sector level. These results show that increased allocation of foreign aid to the health sector not only ameliorates overall health outcomes, but it also improves gender-specific health outcomes. Specifically, maternal mortality appears to significantly decline as more aid is allocated to the health sector. We find similar results for education. An increase in total aid inflows and the share of aid to education in total aid contribute to improving overall educational outcomes as well as gender-specific outcomes. In particular, increased aid allocation to the education sector is associated with a reduction in the gender gap in youth literacy.

It is now widely accepted that reductions in gender inequality can lead to significant improvements in the well-being of children and in overall household welfare. To the extent that aid helps to reduce gender inequality in education and health outcomes, targeting foreign aid towards gender equity in social development outcomes can be a good strategy for improving household well-being. The results also suggest that an increase in the share of the government budget allocated to education and health not only improves overall human development, but it also ameliorates gender-specific development outcomes. In particular, higher government investment in education reduces the gender gap in youth literacy, while increased spending on the health sector in general and on health infrastructure (clean drinking water and modern sanitation) reduces maternal mortality. High age dependency is a drag on overall human development as well as on gender-specific development outcomes. Furthermore, the evidence shows substantial cross-regional variations, especially with SSA and South Asia performing relatively worse than other regions in social and human development.

The importance of public expenditure on health and infrastructure suggests that there is a need to increase domestic financial capacity to finance social sector expenditures. Aid is a significant source of financing for public expenditure in these sectors. However, governments can make even faster progress towards achieving human development and gender equity goals if they can mobilize additional domestic resources to complement aid allocation to social sectors.

The findings from this study have important policy implications for the ability of developing countries to accelerate progress towards national development goals including the MDGs. In particular, the evidence suggests that developing countries are likely to reap substantial 
benefits from increased targeted allocation of foreign aid to health, education and social infrastructure, especially clean drinking water and improved sanitation. Moreover, effective targeting of development and increased access by girls and women to social services and infrastructure are effective strategies for not only accelerating overall human development but also for improving gender equity.

\section{References}

Adato, M. and J. Hoddinott (2007). 'Conditional Cash Transfer Programs: A Magic Bullet for Reducing Poverty?'. 2020 Focus Brief on the World's Poor and Hungry People. Washington, DC: IFPRI.

Agenor, P., O. Canuto, and L. da Silva (2010). 'On Gender and Growth: The Role of Intergenerational Health Externalities and Women's Occupational Constraints'. World Bank Policy Research Working Paper. Washington, DC: World Bank.

Berik, G. and Y. Rodgers (2008). 'Engendering Development Strategies and Macroeconomic Policies: What's Sensible?'. In G. Berik, Y. Rodgers and S. Seguino (eds), Social Justice and Gender Equality: Rethinking Development Strategies and Macroeconomic Policies. London: Routledge.

Berik, G., Y. Rodgers, and S. Seguino (2009). 'Feminist Economics of Inequality, Development and Growth'. Feminist Economics, 15(3): 1-33.

Boone, P. (1996). 'Politics and the Effectiveness of Foreign Aid'. European Economic Review, 40(2): 289-329.

Braunstein, E. (2008). 'The Feminist Political Economy of the Rent-Seeking Society: An Investigation of Gender Inequality and Economic Growth’. Journal of Economic Issues, 42(4): 959-79.

Braunstein, E. (2012). 'Neoliberal Development Macroeconomics: A Consideration of its Gendered Employment Effects'. UNRISD Research Paper 2012-1,

Burnside, C. and D. Dollar (2000). ‘Aid, Policies and Growth’. American Economic Review, 90(4): 847-68.

Clemens, M., S. Radelet, and R. Bhavnani (2004). 'Counting Chickens When They Hatch, The Short-term Effect of Aid on Growth'. Working Paper, Center for Global Development

Collier, P. and D. Dollar (2004). 'Development Effectiveness: What Have We Learnt?'. The Economic Journal, 114(496): F244-71.

Deere, C. D., P. Antrobus, L. Bolles, and E. Melendez (1990). In the Shadow of the Sun: Caribbean Developemnt Alternatives and US Policy. Boulder, CO: Westview Press.

Dreher, A., P. Nunnenkamp, and R. Thiele (2008). 'Does Aid for Education Educate Children?’. World Bank Economic Review, 22(2): 291-314.

Easterly, W. (2006). The White Man's Burden: Why the West's Efforts to Aid the Rest Have Done So Much Ill and So Little Good. New York: Penguin Press.

Elson, D. and N. Cagatay (2000). 'The Social Content of Macroeconomic Policies'. World Development, 28(7): 1347-64. 
Floro, M. (1995). 'Economic Restructuring, Gender and the Allocation of Time'. World Development, 23(11): 1919-26.

Gormanee, K., O. Morrissey, P. Mosley, and A. Verschoor (2005a). 'Aid, Government Expenditure and Aggregate Welfare'. World Development, 33(3): 355-70.

Gormanee, K., O. Morrissey, P. Mosley, and A. Verschoor (2005b). 'Aid, Public Spending and Human Welfare: Evidence from Quantile Regressions'. Journal of International Development, 17(3): 299-309.

Hansen, H. and F. Tarp (2000). 'Aid Effectiveness Disputed'. Journal of International Development, 12(3): 375-98.

Hansen, H. and F. Tarp (2001). 'Aid and Growth Regressions'. Journal of Development Economics, 64(2): 547-70.

Kalaitzidakis, P., T.P. Mamuneas, A. Savvides, and T. Stengos (2001). 'Measures of Human Capital and Nonlinearities in Economic Growth'. Journal of Economic Growth, 6(3): 229-54.

Klasen, S. (2002). 'Low Schooling for Girls, Slower Growth for All? Cross-country Evidence on the Effect of Gender Inequality in Education on Economic Development'. World Bank Economic Review, 16(3): 345-73.

Klasen, S. and F. Lamanna (2009). 'The Impact of Gender Inequality in Education and Employment on Economic Growth: New Evidence for a Panel of Countries'. Feminist Economics, 15(3): 91-132.

Michalowa, K., and A. Weber (2006). 'Aid Effectivness Reconsidered: Panel Data Evidence for the Education Sector'. Discussion Paper: Hamburg Institute of International Economics.

Mishra, P. and D. Newhouse (2009). 'Does Health Aid Matter?'. Journal of Health Economics, 28(4): 855-72.

Ndikumana, L. (2012a). 'Appliquer l'évaluation à l'aide au développement : une solution pour combler le fossé micro-macro de l'efficacité de l'aide?'. Revue d'économie du développement, 2012/4(26): 125-53.

Ndikumana, L. (2012b). 'Applying Evaluation to Development and Aid: Can Evaluation Bridge the Micro-macro Gaps in Aid Effectiveness?'. In F. Bourguigno, M.A. Clemens, J. Mirrlees, J.-D. Naudet, L. Ndikumana, J. Nelson, C. Paradeise, R. Ruben and M. Székely (eds), Evaluation and Its Discontents: Do We Learn from Experience in Development?. Paris: Agence Française de Développement.

Rajan, R. and A. Subramanian (2005). 'What Undermine Aid's Impact on Growth?'. IMF Working Paper.

Richey, L. (2000). 'Gender Equality and Foreign Aid'. In F. Tarp and P. Hjertholm (eds), Foreign Aid and Development, Lesson Learnt and Directions for the Future. London: Routledge.

Seguino, S. (2008). 'Micro-Macro Linkages between Gender, Development and Growth: Implications for the Caribbean Region'. Journal of Eastern Caribbean Studies, 33(4): 842.

Skoufias, E. and B. McCafferty (2001). 'Is Progress Working? Summary of the Results of an IFPRI Evaluation?’. FCND Discussion Paper. 
Stotsky, J. (2006). 'Gender and Its Relevance to Macroeconomic Policy: A Survey'. IMF Working Paper.

World Bank (2011). World Development Report 2012: Gender Equality and Development. Washington, DC: The World Bank.

Xu, Z. (2007). 'A Survey of Intra-Household Models and Evidence'. Munich RePEc Archives. 
Table A1: Summary statistics: full sample and by region

\begin{tabular}{|c|c|c|c|c|c|c|c|c|}
\hline \multirow[t]{2}{*}{ Variable } & \multicolumn{2}{|c|}{ Sample } & \multicolumn{2}{|c|}{$\begin{array}{l}\text { Sub-Saharan } \\
\text { Africa }\end{array}$} & \multicolumn{2}{|c|}{ South Asia } & \multicolumn{2}{|c|}{ Latin America } \\
\hline & Mean & $\begin{array}{l}\text { Std. } \\
\text { Dev. }\end{array}$ & Mean & $\begin{array}{l}\text { Std. } \\
\text { Dev. }\end{array}$ & Mean & $\begin{array}{l}\text { Std. } \\
\text { Dev. }\end{array}$ & Mean & $\begin{array}{l}\text { Std. } \\
\text { Dev. }\end{array}$ \\
\hline $\mathrm{HDI}$ & 0.523 & 0.155 & 0.398 & 0.123 & 0.427 & 0.123 & 0.627 & 0.091 \\
\hline $\begin{array}{l}\text { Change in HDI (1973- } \\
\text { 2010) }\end{array}$ & 0.099 & 0.071 & 0.078 & 0.059 & 0.147 & 0.073 & 0.115 & 0.056 \\
\hline Gender inequality index & 0.524 & 0.137 & 0.622 & 0.085 & 0.588 & 0.112 & 0.489 & 0.083 \\
\hline Education index & 0.474 & 0.186 & 0.349 & 0.151 & 0.351 & 0.151 & 0.568 & 0.125 \\
\hline Health index & 0.664 & 0.158 & 0.507 & 0.117 & 0.628 & 0.138 & 0.784 & 0.085 \\
\hline $\begin{array}{l}\text { Female/Male youth } \\
\text { literacy ratio }\end{array}$ & 89.505 & 16.554 & 80.758 & 20.195 & 83.363 & 19.673 & 99.629 & 4.013 \\
\hline Maternal mortality & 214.785 & 293.638 & 528.915 & 359.813 & 319.010 & 354.511 & 126.147 & 165.159 \\
\hline Total aid/GDP & 6.886 & 19.245 & 11.373 & 27.376 & 2.737 & 3.655 & 3.082 & 10.695 \\
\hline Aid to education/GDP & 0.521 & 1.592 & 0.687 & 1.594 & 0.288 & 0.591 & 0.208 & 0.968 \\
\hline Aid to health/GDP & 0.379 & 1.176 & 0.586 & 1.402 & 0.127 & 0.268 & 0.099 & 0.372 \\
\hline $\begin{array}{l}\text { Aid to education/Total } \\
\text { aid }\end{array}$ & 0.075 & 0.120 & 0.059 & 0.098 & 0.067 & 0.113 & 0.065 & 0.095 \\
\hline Aid to health/Total aid & 0.045 & 0.088 & 0.039 & 0.054 & 0.043 & 0.093 & 0.045 & 0.102 \\
\hline Public health exp/GDP & 3.152 & 2.222 & 2.784 & 2.047 & 2.475 & 1.357 & 3.578 & 1.726 \\
\hline $\begin{array}{l}\text { Public health exp/Total } \\
\text { exp }\end{array}$ & 10.120 & 4.592 & 9.481 & 4.766 & 9.297 & 5.531 & 12.075 & 4.470 \\
\hline $\begin{array}{l}\text { Public education } \\
\text { exp/GDP }\end{array}$ & 3.767 & 1.769 & 3.749 & 1.582 & 3.753 & 1.389 & 3.105 & 1.427 \\
\hline Age dependency ratio & 78.169 & 17.737 & 88.750 & 11.927 & 79.462 & 14.177 & 75.641 & 14.898 \\
\hline $\begin{array}{l}\text { Sanitation access (\% of } \\
\text { Pop) }\end{array}$ & 59.295 & 30.826 & 34.859 & 24.192 & 51.975 & 26.254 & 72.785 & 22.715 \\
\hline $\begin{array}{l}\text { Water access (\% of } \\
\text { Pop) }\end{array}$ & 77.557 & 19.564 & 63.990 & 19.089 & 77.725 & 21.978 & 84.569 & 13.514 \\
\hline Total literacy rate & 72.093 & 23.254 & 56.891 & 24.343 & 62.373 & 23.623 & 85.659 & 13.169 \\
\hline Corruption index & 0.422 & 0.173 & 0.402 & 0.180 & 0.375 & 0.149 & 0.436 & 0.157 \\
\hline $\begin{array}{l}\text { Government stability } \\
\text { index }\end{array}$ & 7.394 & 2.332 & 7.191 & 2.494 & 6.785 & 2.500 & 6.957 & 2.055 \\
\hline Initial HDI & 0.463 & 0.148 & 0.356 & 0.114 & 0.345 & 0.120 & 0.565 & 0.089 \\
\hline
\end{tabular}

Source: Authors' computation from OECD-DAC and World Bank databases (see Table A2). 
Table A2: Variable definitions and sources

\begin{tabular}{|c|c|c|}
\hline Variable and definition & Source & Coverage \\
\hline Human Development Index & $\begin{array}{l}\text { UNDP International Human } \\
\text { Development Indicators }\end{array}$ & $1980-2010$ \\
\hline Change in HDI (1973-2010) & Author's calculations & \\
\hline $\begin{array}{l}\text { Gender Inequality Index (A composite measure reflecting } \\
\text { inequality in achievements between women and men in } \\
\text { three dimensions: reproductive health, empowerment and } \\
\text { the labour market.) }\end{array}$ & $\begin{array}{l}\text { UNDP International Human } \\
\text { Development Indicators }\end{array}$ & $1995-2010$ \\
\hline $\begin{array}{l}\text { Education Index (Composite index based on mean years of } \\
\text { schooling (of adults) and expected years of schooling (of } \\
\text { children). }\end{array}$ & $\begin{array}{l}\text { UNDP International Human } \\
\text { Development Indicators }\end{array}$ & $1980-2010$ \\
\hline $\begin{array}{l}\text { Health Index (Life expectancy at birth expressed as an index } \\
\text { using a minimum value of } 20 \text { years and observed maximum } \\
\text { value over 1980-2010.) }\end{array}$ & $\begin{array}{l}\text { UNDP International Human } \\
\text { Development Indicators }\end{array}$ & $1980-2010$ \\
\hline $\begin{array}{l}\text { Youth Literacy: Ratio of Young Literate Females to Males (\% } \\
\text { ages 15-24) }\end{array}$ & World Development Indicators & $1975-2010$ \\
\hline $\begin{array}{l}\text { Maternal Mortality Ratio (National Estimate, per 100,000 live } \\
\text { births) }\end{array}$ & World Development Indicators & $1975-2010$ \\
\hline Total Disbursement of Aid as Share of GDP (constant USD) & $\begin{array}{l}\text { OECD DAC, WDI and authors' } \\
\text { calculations }\end{array}$ & $1973-2010$ \\
\hline $\begin{array}{l}\text { Total Disbursement of Aid to Education Sector as Share of } \\
\text { GDP (constant USD) }\end{array}$ & $\begin{array}{l}\text { OECD DAC, WDI and authors' } \\
\text { calculations }\end{array}$ & $1973-2010$ \\
\hline $\begin{array}{l}\text { Total Disbursement of Aid to Health Sector as Share of GDP } \\
\text { (constant USD) }\end{array}$ & $\begin{array}{l}\text { OECD DAC, WDI and authors' } \\
\text { calculations }\end{array}$ & $1973-2010$ \\
\hline Share of Total Aid Disbursement going to Education & Authors' calculations & $1973-2010$ \\
\hline Share of Total Aid Disbursement going to Health & Authors' calculations & $1973-2010$ \\
\hline Public Health Expenditure as Share of GDP & World Development Indicators & $1995-2010$ \\
\hline $\begin{array}{l}\text { Public Health Expenditure as Share of Government } \\
\text { Expenditure }\end{array}$ & World Development Indicators & $1995-2010$ \\
\hline Public Spending on Education as Share of GDP & $\begin{array}{l}\text { World Development Indicators } \\
\text { and authors' calculations }\end{array}$ & $1970-2010$ \\
\hline Age Dependency Ratio & World Development Indicators & $1973-2010$ \\
\hline $\begin{array}{l}\text { Percentage of Population with Access to Improved } \\
\text { Sanitation Facilities }\end{array}$ & World Development Indicators & $1990-2010$ \\
\hline $\begin{array}{l}\text { Percentage of Population with Access to Improved Water } \\
\text { Source }\end{array}$ & World Development Indicators & 1990-2010 \\
\hline Adult Literacy Rate (\% of people aged 15 and above) & World Development Indicators & $1975-2010$ \\
\hline Corruption Index & ICRG & $1984-2010$ \\
\hline Government Stability Index & ICRG & $1984-2010$ \\
\hline Initial HDI & Author's calculations & \\
\hline
\end{tabular}

